\title{
Patterns of Synchrony in Neural Networks with Spike Adaptation
}

\author{
C. van Vreeswijk $\dagger^{*}$ and D. Hanselfł \\ $\nmid$ Racah Institute of Physics and Center for Neural Computation, \\ Hebrew University, Jerusalem, 91904 Israel \\ $\ddagger$ Centre de Physique Théorique UMR7466 CNRS \\ Ecole Polytechnique 91128 Palaiseau Cedex, France
}

\begin{abstract}
We study the emergence of synchronized burst activity in networks of neurons with spike adaptation. We show that networks of tonically firing adapting excitatory neurons can evolve to a state where the neurons burst in a synchronized manner. The mechanism leading to this burst activity is analyzed in a network of integrate-and-fire neurons with spike adaptation. The dependence of this state on the different network parameters is investigated, and it is shown that this mechanism is robust against inhomogeneities, sparseness of the connectivity, and noise. In networks of two populations, one excitatory and one inhibitory, we show that decreasing the inhibitory feedback can cause the network to switch from a tonically active, asynchronous state to the synchronized bursting state. Finally, we show that the same mechanism also causes synchronized burst activity in networks of more realistic conductance based model neurons.
\end{abstract}

\footnotetext{
* present address: Gatsby Computational Neuroscience Unit, University College London, 17 Queen Square, London WC1N 3AR, UK.; e-mail: carl@gatsby.ucl.ac.uk

${ }^{\dagger}$ present address: Neurophysique et physiologie du système moteur, EP1848 CNRS, Ecole de Medecine, 45 Rue des Saints Pères 75005, Paris, France; e-mail: David.Hansel@biomedicale.univ-paris5.fr
} 


\section{Introduction}

The Central Nervous System (CNS) displays a wide spectrum of macroscopic, spatially synchronized, rhythmic activity patterns, with frequencies ranging from $0.5 \mathrm{~Hz}(\delta$ rhythm), to $40-80 \mathrm{~Hz}$ ( $\gamma$ rhythm) and even up to $200 \mathrm{~Hz}$ (for a review see Gray, 1994). Rhythmic activities in the brain can also differ by the strength, i.e., by the amplitude of the cross-correlation (CC) peaks (properly normalized) and the precision of the synchrony that can be characterized by the width of the $\mathrm{CC}$ peaks. In many cases, for instance in the visual cortex during visual stimulation, the $\mathrm{CC}$ peaks are narrow, i.e., action potentials of different neurons are correlated across the time scale of the spikes. In other cases, in epileptic seizures in the hippocampus (Silva et al. 1991, Flint and Connors 1996), or in slow cortical rhythms (Steriade et al. 1993), for example, the synchrony which is observed does not occur on a spike to spike basis, but rather on a longer time scale. In these cases, spikes are not synchronized, but the firing rates of the neurons display synchronous modulation, and the firing rate of the neurons can be substantially higher than the frequency of the synchronized rhythmic activity pattern. The mechanisms involved in the emergence of these rhythms remain a matter of debate. An important issue is the respective contribution, in their emergence, of the cellular, synaptic and architectonic properties.

Recent theoretical works have shown that synaptic excitation alone can hardly explain the occurrence of synchrony of neural activity (Hansel et al., 1993, Abbott and Van Vreeswijk, 1993, Van Vreeswijk et al., 1994, Hansel et al. 1995, Gerstner, van Hemmen, and Cowan, 1996). This has lead to the suggestion that inhibition plays an important role in neural synchrony (Hansel et al, 1993, Abbott and Van Vreeswijk, 1994, Hansel et al., 1995, van Vreeswijk 1996). This scenario and its robustness to heterogeneities in intrinsic properties of neurons, to noise and to sparseness of the connectivity pattern has been investigated in detail (White et al., 1997, Chow, 1998, Neltner et al., 1999, Golomb and Hansel, 1999). Neltner et al. (1999) have shown that neural activity in heterogeneous networks of inhibitory neurons can be synchronized at firing rates as large as $150-200 \mathrm{~Hz}$ provided that the connectivity of the network is large, the synaptic interactions and the external inputs are sufficiently strong. This scenario which relies on a balancing between excitation and inhibition can be generalized to networks consisting of two populations of neurons, one excitatory and the other inhibitory (Neltner et al., unpublished). Recent experimental studies in hippocampal slices (Jeffreys et al., 1995); in which excitation has been blocked pharmacologically also support the role of inhibition in neural synchrony. However, other experiments in hippocam- 
pal slices, with carbachol, show that when inhibition is blocked, synchronized burst activity emerges. Modeling studies (Traub et al., 1992) show that the AHP currents may play an important role in the emergence of this burst activity.

Bursting neuronal activity is also observed in the CNS under physiological conditions. For a long time it has been known that the neurons in primary visual cortex often fire bursts of action potentials (Hubel 1959). Evidence has been found (Cattaeno et al., 1981) that the tuning curves to orientation of complex cells in V1 are more selective when computed only from the spikes fired in bursts than when all of the spikes are included. More recent studies (DeBusk et al., 1997, Snider et al., 1998) have also shown that a substantial fraction of the spikes fired by cortical neurons during information processing, occurs during bursts, and have suggested that these spikes play a crucial role in information processing in cortex.

Since slow potassium currents are wide-spread in neurons, it is important to undetstand how they contribute to the shaping of the spatiotemporal patterns of activity. Here we examine how rhythmic activity emerges in a network where the excitatory neurons possess an AHP current responsible for spike adaptation. We study, analytically and numerically, networks of neurons whose excitatory population exhibit spike adaptation. We show that the positive feedback, due to the excitatory synaptic interactions, and the negative and local feedback, due to the spike adaptation, cooperate in the emergence of a collective state in which neurons fire in bursts. These bursts are synchronized across the network but the individual spikes are not. They result from a network effect, since in our model, neurons can fire only tonically when isolated from the network. This state is robust against noise, heterogeneity in the intrinsic properties of the neurons, and sparseness in the connectivity pattern of the network.

The paper is organized as follows: In the next section we introduce the integrate-and-fire (IF) model we study. The properties of the single excitatory neuron dynamics of this model are described in section 3 . In section 4, we study networks that consist only of excitatory neurons. We study the phase diagram in subsection 4.1. The mechanism and properties of the synchronized bursting state are analyzed in the limit of slow adaptation in subsections 4.2 and 4.3, respectively. The next subsection shows that the mechanism is robust to the introduction of inhomogeneities, sparseness of the coupling and noise, and in section 4.5, networks with realistic adaptation time constants are considered. In section 5, we discuss the effect of adding an inhibitory population.

It has been shown recently, that integrate-and-fire networks can differ substantially in their collective behavior, from networks of neurons modeled in a more realistic way in terms of ionic conductances. Given this, in section 6, we show by 
numerical simulations how the results we have obtained for IF networks extend to networks with conductance based dynamics. Finally, we briefly discuss the main results of this work.

\section{Integrate-and-fire model network}

The network model consists of two populations of integrate-and-fire (I\&F) neurons, one is excitatory (E) and the other is inhibitory (I). The excitatory population shows spike adaptation, the inhibitory one does not. The pattern of connectivity is characterized by a matrix, $J_{i j}^{\alpha \beta}, i, j=1, \ldots, N, \alpha=E, I: J_{i j}^{\alpha \beta}=1$, if neuron $j$, in population $\alpha$ makes a synapse on the postsynaptic neuron $i$, from population $\beta$, and $J_{i j}^{\alpha \beta}=0$ otherwise. For the sake of simplicity, we assume that all of the synapses have the same strength and the same synaptic time constants. We neglect propagation delays.

Each excitatory neuron, $i$, is characterized at time, $t$, by its membrane potential, $V_{i}^{E}(t)$, by its adaptation current, $A_{i}^{E}(t)$ and by the total synaptic current into it, $E_{i}^{E}(t)$. These quantities are following the dynamical equations:

$$
\tau_{E} \frac{d V_{i}^{E}}{d t}=I_{i}^{E}-V_{i}^{E}+E_{i}^{E}-A_{i}^{E}
$$

and

$$
\tau_{A} \frac{d A_{i}^{E}}{d t}=-A_{i}^{E}
$$

If, at time $t, V_{i}^{E}$ reaches 1 , a spike occurs, $V_{i}^{E}$ is instantaneously reset to 0 and the adaptation current, $A_{i}^{E}$, is increased by $g_{A} / \tau_{A}$, i.e., $V_{i}^{E}\left(t^{+}\right)=0$ and $A_{i}\left(t^{+}\right)=$ $A_{i}\left(t^{-}\right)+g_{A} / \tau_{A}$.

Similarly, the activity of inhibitory neuron, $i$, is characterized by its membrane potential, $V_{i}^{I}$, and by the total synaptic current into it, $E_{i}^{I}(t)$. The membrane potential satisfies

$$
\tau_{I} \frac{d V_{i}^{I}}{d t}=I_{i}^{I}-V_{i}^{I}+E_{i}^{I}
$$

supplemented with the reset condition, $V_{i}^{I}\left(t^{+}\right)=0$, if the membrane potential of inhibitory neuron, $i$, reached threshold at time $t$.

The external inputs into the excitatory and inhibitory neurons are $I_{i}^{E}$ and $I_{i}^{I}$, respectively. The membrane time constant for the inhibitory cells is $\tau_{I}$ (for simplicity, we assume that all of the neurons in the same population have the same membrane time constant). Note that we are not including the adaptation current 
in the dynamics of the inhibitory neurons in order to account for the fact that in cortex, most of the inhibitory neurons do not display spike adaptation (Connors et al., 1982, Connors and Gutnick, 1990, Ahmed et al., 1998).

For the excitatory neurons, we will assume a typical value for the membrane time constant of $\tau_{E}=10 \mathrm{msec}$. In the following, time will be measured normalized to $\tau_{E}$, so that $\tau_{E}$ will be omitted from the equations.

The synaptic currents, $E_{i}^{E}$ and $E_{i}^{I}$, into excitatory and inhibitory cell, $i$, are given respectively by:

$$
E_{i}^{\alpha}=\sum_{\beta=E, I} \sum_{j, k} \frac{1}{\tau_{1 \beta}-\tau_{2 \beta}} J_{i j}^{\alpha \beta} g_{\alpha \beta}\left(e^{-\left(t-t_{j, k}^{\beta}\right) / \tau_{1 \beta}}-e^{-\left(t-t_{j, k}^{\beta}\right) / \tau_{2 \beta}}\right)
$$

where $J_{i j}^{\alpha \beta}$ is the connectivity matrix of the network, $\alpha=E, I, \tau_{1 \beta}$ and $\tau_{2 \beta}$ are the rise and decay times of the synapses projecting from population $\beta, t_{j k}^{\beta}$ denotes the $k$ th time neuron $j$ of population $\beta$ fired an action potential, and $g_{\alpha \beta}$, is the strength of the synapses projecting from population $\beta$ to population $\alpha$. Note that $g_{E E}$ and $g_{I E}$ are positive, while $g_{E I}$ and $g_{I I}$ are negative. In most of the work we will use a synaptic decay and rise times of $\tau_{1, E}=3 \mathrm{msec}$ and $\tau_{2, E}=1 \mathrm{msec}, \tau_{1, I}=6 \mathrm{msec}$ and $\tau_{2, I}=1 \mathrm{msec}$, respectively. Most of the paper deals with network with allto-all connectivity. In that case, the connectivity matrix is $J_{i j}^{\alpha \beta}=1-\delta_{i, j} \delta_{\alpha, \beta}$ and $g_{\alpha \beta}=G_{\alpha \beta} / N$, where $\delta_{i, j}=1$ for $i=j$ and $\delta_{i, j}=0$ otherwise, and similar for $\delta_{\alpha, \beta}$. We assume that $G_{\alpha \beta}$ is independent of $N$.

For simplicity, we have assumed that the synapses and the adaptation are described by currents rather than conductance changes. Describing these variables by conductance changes does not qualitatively affect the result of our analysis.

In numerical simulations of the network, Eqns. (1-4) were integrated using a second order Runge-Kutta method supplemented by a first order interpolation of the firing time at threshold as explained in (Hansel et al., 1998, Shelley, 1999). This algorithm is optimal for I\&F neuronal networks and allows us to get reliable results for time steps, $\Delta t$, which are substantially large. The integration time step is $\Delta t=0.25 \mathrm{~ms}$. Stability of the results was checked against varying the number of time steps in the simulations and their size. When burst duration and interburst interval were measured, we discarded the first few bursts to eliminate the effects of the initial values and averaged these quantities over 10 to 20 bursts. 

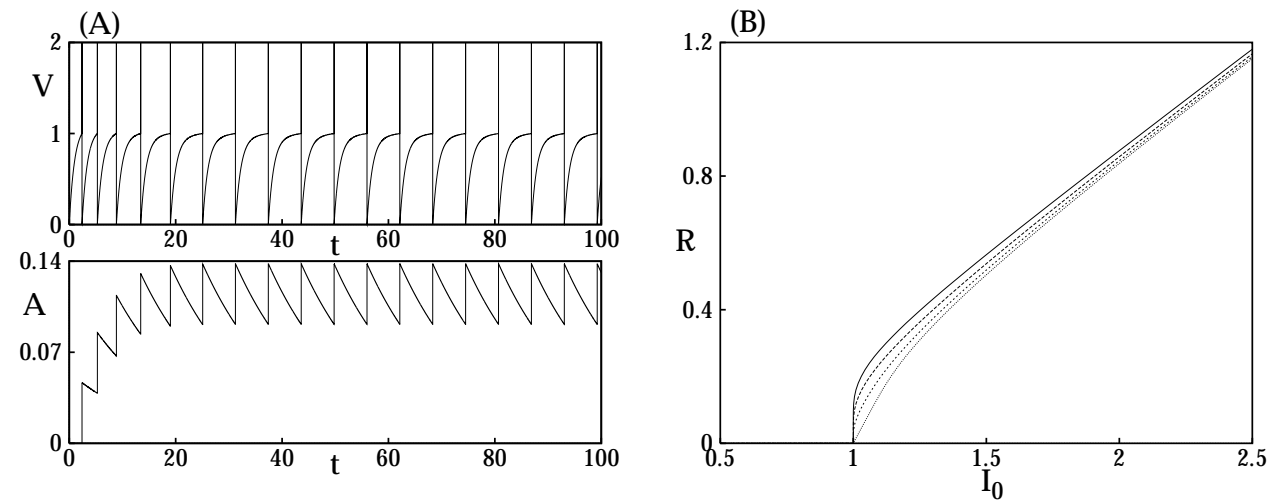

Figure 1: A: Response of a single cell to current injection. At, $t=0$, a constant current, $I_{0}=1.1$, is applied. Upper Panel: The neuron voltage. Spikes were added at the times neuron when the voltage crosses the threshold, $\theta=1$. Lower Panel: The adaptation current. B: The firing frequency of the single model neuron with $g_{A}=0.675$ versus the constant applied current. The rate is expressed in units of $1 / \tau_{m}$. With $\tau_{m}=10 \mathrm{msec}, R=1$ corresponds to a rate of $100 \mathrm{~Hz}$. Solid line: $\tau_{A}=1$, long dashed line: $\tau_{A}=2$, short dashed line: $\tau_{A}=5$, dotted line: $\tau_{A}=\infty$.

\section{Single neuron properties of the excitatory popula- tion}

Before we study the activity of the model network, we first describe the single cell characteristics of the excitatory neurons in the presence of an external stimulus, $I_{0}$. In the single neuron study Figure $1 \mathrm{~A}$ shows the response of a single cell to constant input. For $t<0, I_{0}=0$ and $V(t)=A(t)=0$. At $t=0$, a constant current, $I_{0}>1$, is turned on and the neuron starts firing. As the adaptation currents build up, the firing rate diminishes. Eventually, the cell stabilizes in a periodic firing state. It can be shown that the decrease of the instantaneous firing rate towards its limit at large time is well approximated by an exponential relaxation. Thus, the single model neurons are tonically firing adaptive neurons.

After a transient period, the cell fires periodically with period, $T$. Assume that the cell fires at time $t_{0}$. Immediately after the spike the adaptation current will be at its maximum value $A_{0}$ and then decrease untill the next spike is fired. Thus $A\left(t_{0}^{+}\right) \equiv A_{0}$. This implies that $A\left(t_{0}+T^{+}\right)=A_{0}$. Therefore, just before the spike at $t_{0}+T, A$ is given by

$$
A\left(t_{0}+T^{-}\right)=A_{0}-g_{A} / \tau_{A} .
$$

On the other hand, since the cell does not spike between times $t=t_{0}$ and $t=$ 
$t_{0}+T, A\left(t_{0}+T^{-}\right)$also satisfies

$$
A\left(t_{0}+T^{-}\right)=A_{0} e^{-T / \tau_{A}} .
$$

Therefore, $A_{0}$ is given by

$$
A_{0}=\frac{g_{A}}{\tau_{A}\left(1-e^{-T / \tau_{A}}\right)} .
$$

Just after the spike at time $t=t_{0}$, the membrane potential of the cell is reset to $0, V\left(t_{0}^{+}\right)=0$. Between spikes, $V$ satisfies

$$
\frac{d}{d t} V=I_{0}-V-A(t)
$$

with $A(t)=A_{0} e^{-\left(t-t_{0}\right) / \tau_{A}}$. Consequently, for $0<t<T, V\left(t_{0}+t\right)$ is given by

$$
V\left(t_{0}+t\right)=I_{0}\left(1-e^{-t}\right)-\frac{A_{0} \tau_{A}}{\tau_{A}-1}\left(e^{-t / \tau_{A}}-e^{-t}\right) .
$$

Since the cell fires again at time $t_{0}+T, V\left(t_{0}+T^{-}\right)=1$, the period $T$ satisfies

$$
I_{0}\left(1-e^{-T}\right)-\frac{g_{A}}{\tau_{A}-1} \frac{e^{-T / \tau_{A}}-e^{-T}}{1-e^{-T / \tau_{A}}}=1 .
$$

Figure $1 \mathrm{~B}$ shows the firing rate at large time, $R=1 / T$, for a single neuron as a function of the input current, $I_{0}$, for different values of the adaptation time constant, $\tau_{A}$. If the input is below threshold, $I_{0}<1$, the cell does not fire. For large input currents, such that $T \ll 1$, we can use Eqn. (10) to approximate the rate as

$$
R=\frac{1}{1+g_{A}}\left(I_{0}-1 / 2\right),
$$

independent of $\tau_{A}$. Therefore, the rate varies linearly with the external input, as is the case for the IF model without adaptation. The effect of the adaptation is to divide the gain of the frequency-current curve by a factor that does not depend on the adaptation time constant.

Just above the threshold the rate increases non-linearly with, $I_{0}$. Using $0<$ $\delta I \equiv I_{0}-1 \ll 1$, one finds from Eqn. (10) that, assuming $\tau_{A}>1$, the rate, $R$, satisfies asymptotically in the limit $T \gg \tau_{A}$ :

$$
R=\frac{1}{\tau_{A}}\left[-\log (\delta I)+\log \left(\frac{g_{A}}{\tau_{A}+1}\right)\right]^{-1} .
$$


So, for any $\tau_{A}>1$, the rate has a $-1 / \log (\delta I)$ dependence on the external input, as is the case for IF neurons without adaptation. However, the prefactor here is $\frac{1}{\tau_{A}}$, whereas when there is no adaptation, the prefactor is 1 (when $g_{A} \rightarrow 0$ a crossover occurs between the two behaviors).

If $\tau_{A}$ is very large, a third regime exists for $1 \ll T \ll \tau_{A}$. In this limit, Eqn. (10) can be written as

$$
\left(I_{0}-g_{A} R\right)\left(1-e^{-1 / R}\right)=1 .
$$

Thus, for low rates, $R \ll 1$, the rate is approximately given by

$$
R=\frac{\delta I}{g_{A}}
$$

In the limit $\tau_{A} \rightarrow \infty$, the logarithmic dependence of $R$ on $\delta I$ becomes negligible due to the small prefactor $1 / \tau_{A}$ and Eqn. (14) extends up to the bifurcation point. Therefore, in this limit, the transfer function starts linearly, with a slope $1 / g_{A}$. The slope stays nearly constant as long as $e^{-1 / R}$ is negligible compared to 1 . For larger $I_{0}$, the slope gradually decreases, and for $I_{0} \gg 1+g_{A}$, the rate depends linearly on $I_{0}$, but with a smaller slope $1 /\left(1+g_{A}\right)$. This is shown in Figure 1B. It should be noted that in our simple model, the transfer function of the neurons can be approximated by one semi-linear function over a large range of frequencies only for $g_{A} \gg 1$. While we find $d R / d I_{0}=1 / g_{A}$ near threshold, for an input that gives a rate $R=1$, corresponding to $100 \mathrm{~Hz}$, the slope changes substantially to $d R / d I_{0} \approx 1 /\left(g_{A}+0.9207\right)$.

\section{One excitatory population with spike adaptation}

\subsection{The phase-diagram of the model}

We now consider the case in which all of the inhibition has been blocked in the model network, focusing on the behavior of the excitatory population. We first consider the case where all of the neurons are identical, all-to-all coupled, with $G_{E E}=g_{s}$, and receive the same external input, $I_{0}$. The case of heterogeneous and sparsely connected networks will be considered later (see section 4.4).

As we saw in the previous section, the single cells fire periodically if they receive constant input. In an asynchronous state, (AS), the firing times of the neurons, are evenly distributed, and the input to the cells is constant (up to fluctuations of order $O(1 / N)$ ). This implies that in a large asynchronous network, the neurons 


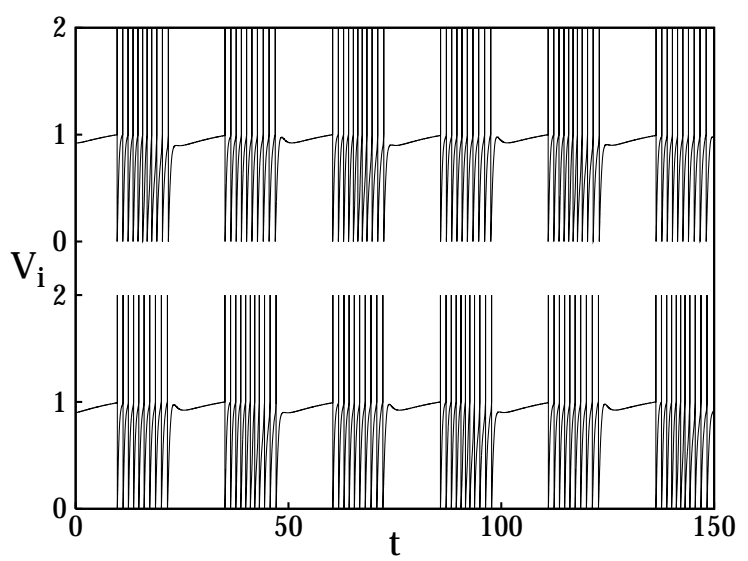

Figure 2: Voltage traces of two neurons in a network that is in the bursting state. Simulation was started at $t=0$ with random initial conditions. Traces are shown after the transient activity has died out. Parameters used: $I_{0}=1.1, g_{A}=0.4, \tau_{A}=150 \mathrm{msec}$ and $g_{s}=0.675$. Time is in units of $\tau_{m}$.

cannot fire in bursts. The stability of the asynchronous state can be determined analytically (Van Vreeswijk, 1999). Here we will only mention the results of this analysis.

There are two ways in which the AS can become unstable.

- For weak external input: When the input, $I_{0}$, is just above the threshold, the AS is stable if the synaptic coupling, $g_{s}$, is sufficiently strong. If $g_{s}$ is decreased, the AS becomes unstable through a normal Hopf bifurcation to a synchronized state in which the cells continue to fire tonically. In this state, neural activity is synchronized on the spike to spike level. This instability is similar to that in networks of IF neurons without adaptation (van Vreeswijk 1996).

- For strong external input: When $I_{0}$ is sufficiently large and the adaptation, $g_{A}$, sufficiently strong, the asynchronous spiking state is stable for small $g_{s}$. When $g_{s}$ is increased, the AS state is destabilized through an inverted Hopf bifurcation. Past this bifurcation, the neurons fire in bursts as shown in Fig. 2. In the following, we focus on this scenario. As we will see, this synchronized state is characterized by a coherent neural activity on the burst to burst level, where spikes are either not synchronized, or weakly synchronized. 


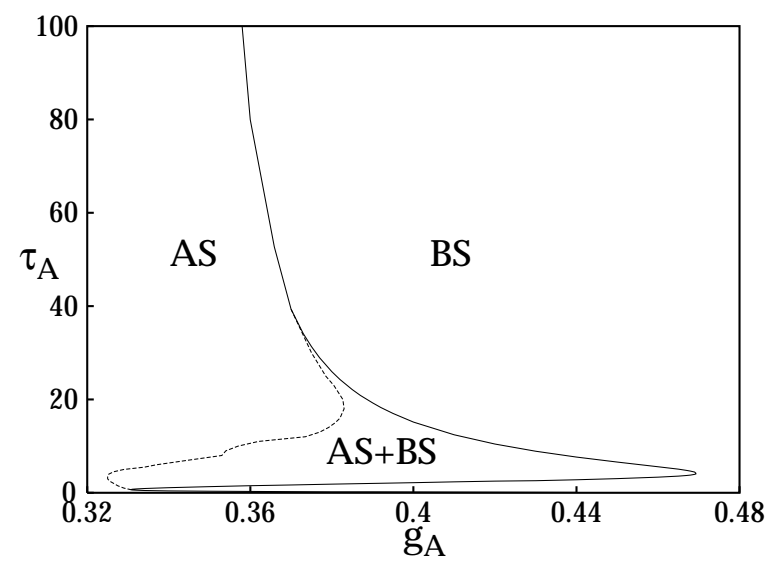

Figure 3: Phase diagram of the integrate-and-fire network with adaptation. Dependence of the final state of the network on $\tau_{A}$ (in units of $\tau_{m}$ ) and $g_{A}$ is shown. Other parameters: $I_{0}=1.1$, $g_{s}=0.675$. AS: The neurons are spiking and the network activity is not synchronized; SB: The neurons are bursting and the network activity is synchronized; AS+SB: In this region the network displays bistability. Depending on the initial condition, the network settles into the asynchronous spiking state or a synchronous bursting state.

A phase diagram of the network is shown in Fig. 3 as a function of $\tau_{A}$ and $g_{A}$. Here we have fixed the parameters $I_{0}$ and $g_{s}$ at values for which the network bursts for sufficiently large $g_{A}$ (second scenario above). There is a region of bistability, in which the network state can evolve to a synchronized bursting state or an asynchronous spiking state depending on the initial conditions. For very slow adaptation the region of bistability is small, but it is considerable for faster adaptation.

\subsection{Mechanism of synchronized bursting in the limit $\tau_{A} \rightarrow \infty$}

As mentioned above, the determination of the stability of the asynchronous state is quite involved. For very slow adaptation however, it is relatively simple to understand the bifurcation to that synchronized bursting state. To see this, let us first recall some of the properties of connected networks of IF neurons without adaptation.

Assuming that the neurons fire asynchronously, it is straightforward to show (see Abbott and van Vreeswijk, 1993) that if the external input, $I_{0}$, is sufficiently large, neurons are firing at constant rate, $R\left(I_{0}\right)$, given by

$$
\left(I_{0}+g_{s} R\right)\left(1-e^{-1 / R}\right)=1
$$




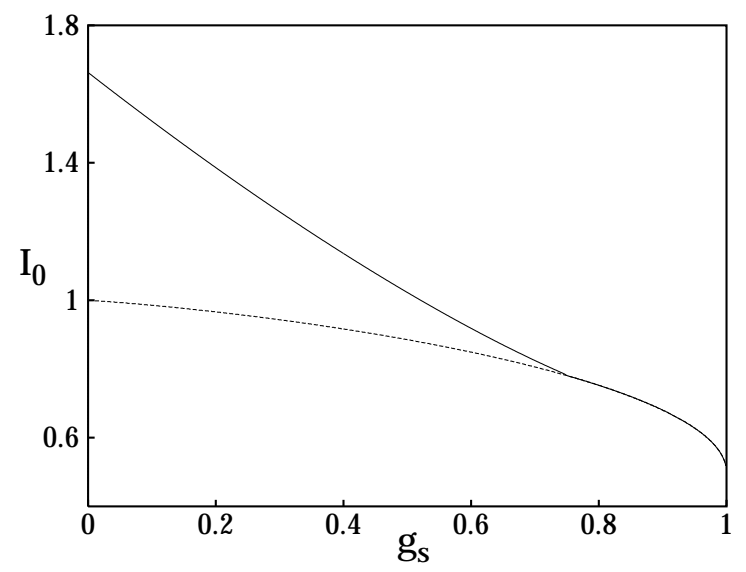

Figure 4: The network without adaptation $\left(g_{A}=0\right)$. Solid line: the critical value, $I_{c}$, of the external current, $I_{0}$, above which the asynchronous state is stable is plotted versus the synaptic strength, $g_{s}$. Dashed line: the minimum value, $I_{\min }$, of the external current, $I_{0}$, below which the asynchronous state does not exist is plotted versus the synaptic strength, $g_{s}$.

According to this equation, the minimal current, $I_{\text {min }}$, required for the neurons to be active in the asynchronous state (AS) depends on the synaptic strength $g_{s}$. As shown in Figure $4, I_{\min }$ ranges from $I_{\min }=0.5$, for $g_{s}=1$, to $I_{\min }=1$, as $g_{s}$ approaches 0 .

Even if the AS state of a neuronal network exists, it is possible that the network does not settle in this state, but in another state, in which the neuronal activity is partially synchronized. For I\&F networks, following (Abbott and van Vreeswijk, 1993), one finds that that there is a critical current $I_{c}>I_{\min }$, above which the AS is stable. This critical current depends on $g_{s}$ and the synaptic time constants, $\tau_{1}$ and $\tau_{2}$. Figure 4 shows the dependence of $I_{c}$ on $g_{s}$. For small $g_{s}$, the critical input exceeds the firing threshold current, $I_{c}>1$. In that case, the bifurcation at $I_{c}$ is a normal Hopf bifurcation leading to a partially synchronized state, in which the average rate of neurons is lower than in the (unstable) AS. This rate goes to 0 as $I_{0}$ approaches 1 . This is shown graphically in Fig $5 \mathrm{~A}$.

When $g_{s}$ is sufficiently large, the critical input is below the threshold current, $I_{c}<1$, see Fig 5B. The network goes through an inverted Hopf bifurcation as $I_{0}$ is reduced past $I_{c}$. For $I_{0}<I_{c}$, the only stable state is the quiescent state (QS), in which the neurons are not firing. The network settles in this state. For $I_{c}<I_{0}<1$, there are two stable states, the AS and the QS. Presumably, there exists also a third state, in which neuronal activity is partially synchronized, with a firing rate which approaches the rate of the AS, when $I_{0} \rightarrow I_{c}$, and approaches 

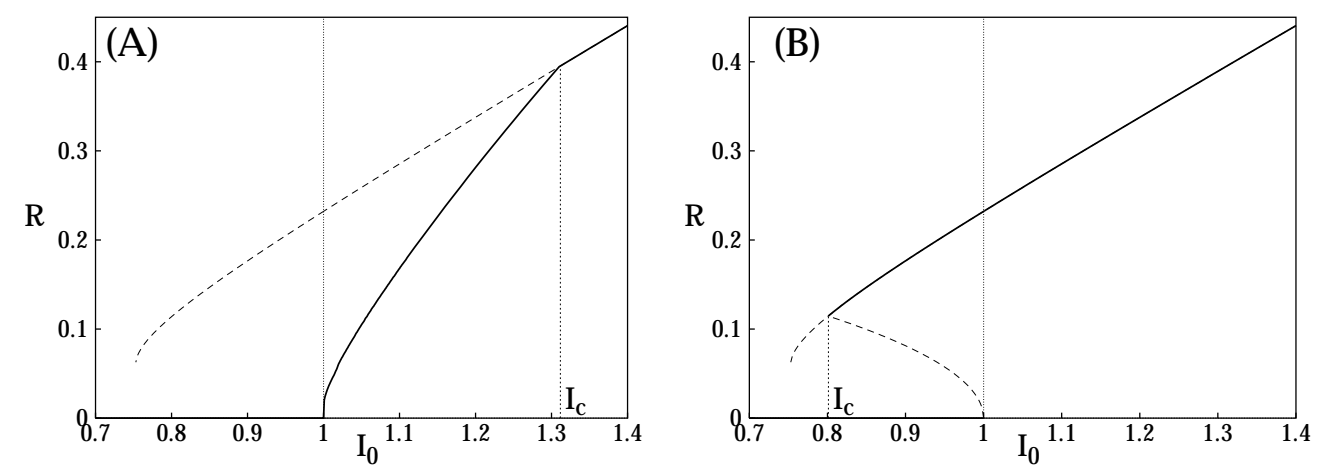

Figure 5: The firing rate of the neurons in the network without adaptation $\left(g_{A}=0\right)$ in the asynchronous state is plotted versus the external current. Solid line: Stable solutions. Dashed lines: Unstable solutions. The critical current $I_{c}$ is indicated on the x-axes. A: $g_{s}=0.3$ : The asynchronous state is unstable below threshold current $\left(I_{0}=1\right)$. B: $g_{s}=0.7$ : The asynchronous state is stable in a range of the external current below threshold.

0 , as $I_{0} \rightarrow 1$. However, one can conjecture that this state is unstable.

In the following, we consider that $g_{s}$ is sufficiently large, so that $I_{c}<1$. If $I_{0}$ is time dependent and decreases slowly (compared to the characteristic time scales of the network dynamics) starting from above threshold, the network settles in the AS. It remains there until $I_{0}$ reaches $I_{c}$, where it abruptly switches to the QS. On the other hand, if $I_{0}$ increases slowly from below $I_{c}$, the network remains in the QS until $I_{0}$ reaches 1, a point at which the neurons start to fire again.

Let us now assume that the neurons display a very slow spike adaptation $\left(\tau_{A} \rightarrow \infty\right)$, and that at time $t=0$ the adaptation and the membrane potential are randomly distributed. For $t>0$, a constant suprathreshold stimulation is applied, i.e., $I_{0}>1$. Since $\tau_{A}$ is large, individual spikes increase the adaptation current $A_{i}$ only by a tiny amount $g_{A} / \tau_{A}$. Therefore after a transient period following the stimulation, $A_{i}(t)$ reflects the cumulative effect for all spikes of neuron $i$ that occurred, roughly, between times $t-\tau_{A}$ and $t$, the timing of these spikes on a time-scale much smaller than $\tau_{A}$ can be neglected in the calculation of $A_{i}$. thus we can write

$$
\tau_{A} \frac{d A_{i}}{d t}=-A_{i}+g_{A} R_{i}(t),
$$

where $R_{i}(t)$ the rate of neuron $i$ smoothed over some window with width much less than $\tau_{A}$. In this case $R_{i}(t)$ is the rate of a $I \& F$ neuron without adaptation that 
receives an input $I_{0}+g_{s} E(t)-A_{i}$. Thus the neurons that start out with the largest $A_{i}(0)$ will be the ones with the lowest rates. As a result their adaptation current will decrease relative to neurons for which $A_{i}(0)$ is smaller, so that after a time which is large compared to $\tau_{A}$ the level of the adaptation current, and therefore the rates, of all cells converges. Thus we can set $A_{i}=A$ and $R_{i}=R$.

If $A<I_{0}-1$ the network is in the AS, with the cells firing at a rate $R\left(I_{0}-A\right)$, $A$ obeys

$$
\tau_{A} \frac{d}{d t} A=-A+g_{A} R\left(I_{0}-A\right) .
$$

For sufficiently large $g_{A}, A$ increases until it reaches $A_{c}=I_{0}-I_{c}$, where the activity of the network drops suddenly to zero, since the only stable state is the QS. In the QS, $A$ decreases, satisfying

$$
\tau_{A} \frac{d}{d t} A=-A
$$

When $A$ has decreased sufficiently (to $A=I_{0}-1$ ), the neurons will again start to fire, rapidly evolving to the AS, and $A$ increases again. Thus, the network switches periodically from the AS to the QS. This is illustrated in Fig. 6. This bursting mechanism requires that the adaptation current, $A$, has to continue to grow, as long as the network is in the AS. Thus, $g_{A}$ has to satisfy

$$
g_{A}>g_{A, c} \equiv \frac{I_{0}-I_{c}}{R\left(I_{c}\right)} .
$$

If $g_{A}$ does not satisfy this constraint, the network reaches an equilibrium with $A=A_{e}=g_{A} R\left(I_{0}-A_{e}\right)$, and the network settles in the AS state.

There is no simple way to determine analytically the behavior of the bursting state for finite $\tau_{A}$. However, numerical simulations confirm that this scenario remains valid for adaptation currents with parameters in a physiological range (Section 4.5). This analysis shows how synchronized bursts can emerge cooperatively from the combination of a strong excitatory feedback with slow and sufficiently strong spike adaptation. In the next section, we study the properties of these bursts.

\subsection{Properties of the Bursting Network in the large $\tau_{A}$ limit}

Equations (17) and (18) suggest that we can calculate the burst duration and the inter-burst interval in the large $\tau_{A}$ limit. Indeed, according to Eqns. (17) and (18) 


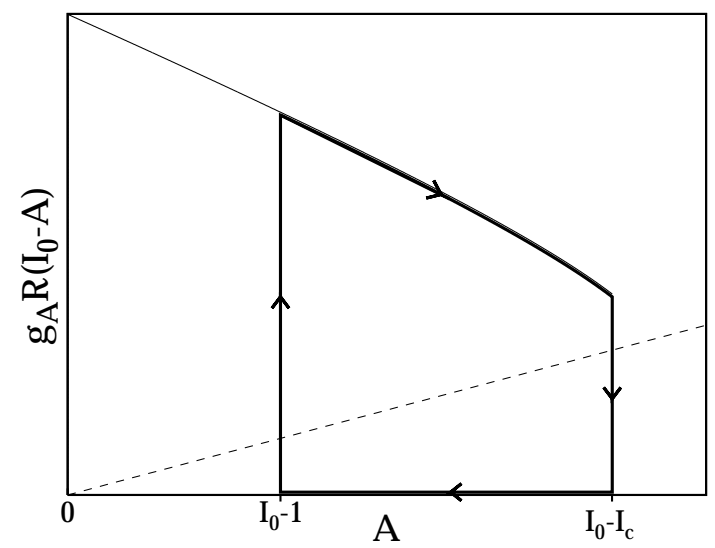

Figure 6: Limit cycle behavior of $A$ in the large $\tau_{A}$ limit. If the network fires asynchronously, $A$ is driven to $g_{A} R\left(I_{0}-A\right.$ ) (solid line). If this quantity is larger than $A$ (dashed line), $A$ increases. When $A$ exceeds $I_{0}-I_{c}$ the network becomes quiescent and $A$ is driven to 0 . When $A$ is less than $I_{0}-1$ the network jumps back to the asynchronous state. $A$ and the rate $R$ periodically traverse a closed trajectory (thick solid line).

the burst duration, $T_{B}$, and the inter-burst interval, $T_{I}$, are given by

$$
T_{B}=\tau_{A} \int_{I_{0}-1}^{I_{0}-I_{c}} \frac{d A}{g_{A} R\left(I_{0}-A\right)-A},
$$

and

$$
T_{I}=\tau_{A} \int_{I_{0}-1}^{I_{0}-I_{c}} \frac{d A}{A}=\tau_{A} \log \left(\frac{I_{0}-I_{c}}{I_{0}-1}\right) .
$$

Thus, both have a duration that is of order $\tau_{A}$. However, there is a subtlety that has to be taken into account. Between bursts, the synaptic feedback is negligible, so that for all cells the membrane potential $V_{i}$ satisfies

$$
\frac{d}{d t} V_{i}=I_{0}-V_{i}-A
$$

This means that between bursts, the difference between the membrane potential decreases. In fact, the standard deviation in potential, $\sigma_{V}$, given by

$$
\left(\sigma_{V}\right)^{2}=N^{-1} \sum_{i} V_{i}^{2}-\left(N^{-1} \sum_{i} V_{i}\right)^{2}
$$

satisfies between bursts

$$
\frac{d}{d t} \sigma_{V}=-\sigma_{V}
$$


as can be seen from considering $d \sigma_{V}^{2} / d t$ and using Eqn. (22). After the burst is terminated, $\sigma_{V}$ is of order 1. Therefore, at the end of the interburst period, $\sigma_{V}$ is of order $e^{-T_{I}}$. For large $\tau_{A}$, this is extremely small. This means that just before the neurons start to fire again, they are very nearly synchronized. However, when the burst has started, the cells are driven away from synchrony (since we have assumed that the synaptic time constants and the synaptic strength are such that excitatory interactions destabilize the synchrony of the network at the spike to spike level.) For a nearly synchronized network, it can be shown that when the network is again active, $\sigma_{V}$ satisfies

$$
\sigma_{V}(T+t)=\sigma_{V}(T) e^{\lambda t}
$$

where $T$ is the time at which the network becomes active again. Here, $\lambda$ depends non-trivially on the network parameters, but is of order 1. Eqn. (25) is valid as long as $\sigma_{V}(T+t)$ is small, for larger $\sigma_{V}$, the standard deviation in the membrane potential grows slower, and it asymptotically goes to $\sigma_{V A}$, the value of $\sigma_{V}$ in the AS, where $\sigma_{V A}$ is of order 1 . Since $\sigma_{V}(T)$ is of order $e^{-T_{I}}$, it will take at least a time of order $T_{I} / \lambda$ (which is of order $\tau_{A}$ ) before $\sigma_{V}$ is of order 1 and the network activity is appreciably desynchronized.

Since the burst duration is also of order $\tau_{A}$, this implies that for a finite fraction of the burst the network is appreciably away from the AS, even in the large $\tau_{A}$ limit. Because, for a given level of external input, a network in a partially synchronized state fires at a lower mean rate than in the asynchronous state, (see Fig. 5B), one expects that Eqn. (20) underestimates the duration of the burst, even in the large $\tau_{A}$ limit. Unfortunately, there is no theoretical expression for the average rate in a network that is partially synchronized. The increase in desynchronization is also not well understood in this state, and it is not possible to take into account the extremely high level of synchrony at the start of the burst, nor to obtain an exact theoretical expression for the burst duration.

The interburst time interval should be correctly predicted from Eqn. (21). This is because, while at the beginning of the burst the cells are nearly synchronized, they fire completely asynchronously at the end of the burst, so the theory in the previous section correctly predicts the state of the neurons when the burst terminates. Since the inter-burst interval depends only on the value of $A$ at the termination of the burst, Eqn. (21) gives the correct value of the inter-burst interval, even though burst duration is not predicted correctly by Eqn. (20).

Figure 7 shows the dependence of $T_{B} / \tau_{A}$ and $T_{I} / \tau_{A}$ on the external input, $I_{0}$, for $\tau_{A}=100$. The quantities $T_{B}$ and $T_{I}$ were determined using numerical 


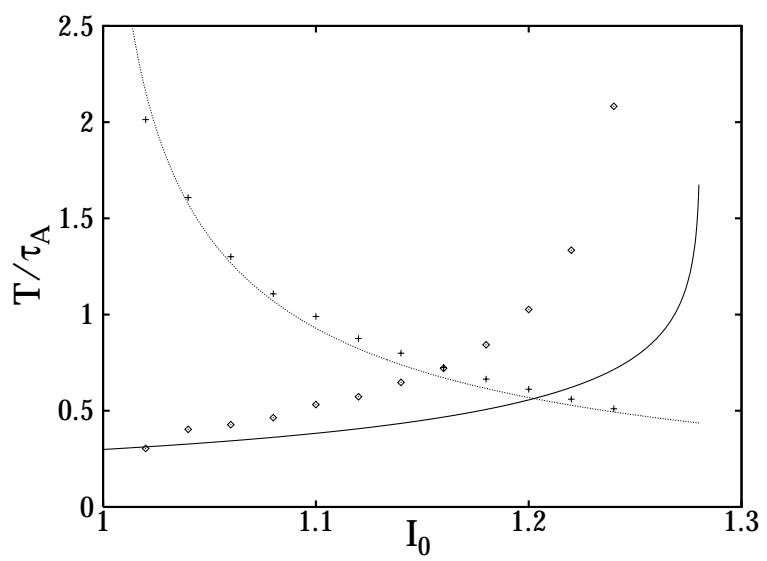

Figure 7: The interburst interval, $T_{I}$, (solid line) and the intraburst duration, $T_{B}$, (dashed line) are plotted versus the external input, $I_{0}$, in the large $\tau_{A}$ limit. Parameters are: $g_{s}=0.675$ and $g_{A}=0.6$. Results from numerical simulations for $\tau_{A}=100$, are also plotted: pluses for $T_{I}$, diamond for $T_{E}$. Numerical integration of the dynamics was performed with a time step, $\Delta t=$ 0.025 . The network consists of $N=100$ excitatory neurons. The synaptic time constant of the synapses were: 0.1 for the rise time and 0.3 for the decay time. For each value of the current a simulation was performed and stopped after 100 bursts have occurred and the burst duration and interburst interval determined by averaging of all but the first 5 bursts.

simulations of a network of $N=100$ neurons. The values predicted by Eqns. (20) and (21) are also shown. The burst duration is underestimated by Eqn. (20). On the other hand, the values of $T_{I}$ predicted by Eqn. (21) are in good quantitative agreement with the simulations.

It is interesting to note that the switching between desynchronizing episodes when the network is active and increasing synchrony during inactive episodes that our model exhibit also shows up in other model networks, for example in Li and Dayan's EI network (Li and Dayan, 1999). In this model the network is driven to inactivity by slowly activating inhibitory units, which are subsequently slowly turned off. Though the mechanism by which their network 'bursts' is very different from ours, their model has, in some parameter regimes, a tendency towards convergence of state variables during the quiescent period and while they diverge when the network is active. This mimics the alternating con- and divergent episodes for the voltages in our network. 


\subsection{Robustness of the bursting mechanism}

So far, we have considered a very idealized network, with all to all coupling, identical neurons, and where each neuron receives the same noiseless external input. Here we show that the mechanism for network bursting described in the previous section is robust to heterogeneities, sparseness of the connectivity, and noise.

Heterogeneities: We consider a network in which the neurons receive heterogeneous external inputs: $I_{i}=I_{0}+\delta I_{i}, i=1, \ldots, N$ (with $\sum_{i} \delta I_{i}=0$ ). Other types of heterogeneities, e.g., in the membrane time constant or in the threshold, can be treated similarly.

The limit of weak level of heterogeneities, $\sigma_{I} \equiv N^{-1} \sum_{i}\left(\delta I_{i}\right)^{2} \ll 1$, can be studied analytically, as shown in Appendix A. We find that in the presence of heterogeneous input, it takes a time of the order of $-\log \left(\left(1-e^{-T_{I} / \tau_{A}}\right)^{2} \sigma_{I}^{2}\right) / \lambda$ before the asynchronous state is reached. So, as long as $-\log \left(\sigma_{I}^{2}\right)$ is large compared to $\tau_{A}$, it takes a negligible fraction of the burst duration before the cells fire asynchronously. For large $\tau_{A}$, the inhomogeneity, $\sigma_{I}$, can be extremely small, so that its effect on $R\left(I_{0}\right)$ and $I_{c}$ can be neglected. Consequently, Eqns. (20) and (21) will predict $T_{B}$ and $T_{I}$ correctly in the limit where $\tau_{A}$ is large, $\sigma_{I}$ small, while $-\log \left(\sigma_{I}^{2}\right) \gg \tau_{A}$. However, an extremely large $\tau_{A}$ is required to check numerically the crossover between the two limits.

For a finite level of heterogeneities, the approximation of Appendix A does not hold. However, one can also generalize the large $\tau_{A}$ approximation of Section 5 to this case. Indeed, in the large $\tau_{A}$ limit, the adaptation, $A_{i}$, of neuron $i$ satisfies the self-consistent equations:

$$
\tau_{A} \frac{d A_{i}}{d t}=-A_{i}+g_{A} r\left(I_{0}+\delta I_{i}+g_{s} \bar{R}-A_{i}\right)
$$

where $r(I)=1 / \log [I /(I-1)]$ for $I>1$, and $r(I)=0$ otherwise, while $\bar{R}$ has to be determined self-consistently from

$$
\bar{R}=\frac{1}{N} \sum_{i=1}^{i=N} r\left(I_{0}+\delta I_{i}+g_{s} \bar{R}-A_{i}\right)
$$

This set of equations can be studied numerically. We have evaluated this system of equations with $N=100$, for $g_{s}=0.6, g_{A}=0.675, I_{0}=1.1$. We have found that the network continues to show synchronous bursting activity even for 


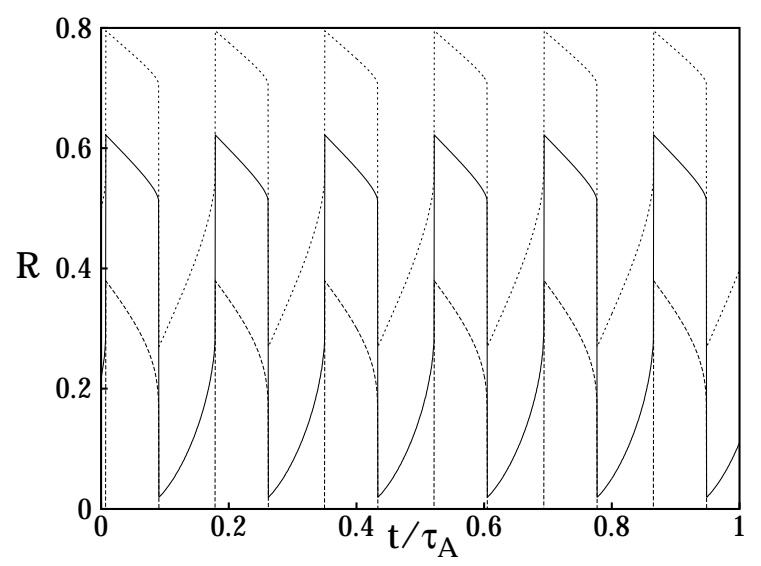

Figure 8: The firing rate in a heterogeneous network in the large $\tau_{A}$ limit, after the transient has died out in a network with 100 neurons. Solid line: Mean rate $\bar{R}$, dashed line: Rate for neuron with input $I_{i}=I_{0}-\delta$, dotted line: Rate for neuron with input $I_{i}=I_{0}+\delta$. Parameters: $I_{0}=1.1$, $g_{s}=0.6, g_{A}=0.675, \delta=0.3$.

values of $\delta$ as large as $\delta=0.3$, that is with input currents $I_{i}$ ranging from $I_{i}=0.8$ to $I_{i}=1.4$. Therefore the bursting state is extremely robust to heterogeneities.

This is shown in Fig. 8. This figure shows the time course of firing rate for the neuron with the largest external input as well as that for the neuron with the smallest input. While the onset of the bursts and the burst duration of these cells are very different, and the firing rates during the bursts vary substantially, both cells still show very clear burst activity. Cells with intermediate inputs show firing rates that are between these two extremes.

If $\delta$ is increased beyond 0.4 the network settles in the AS. In the intermediate region, $0.3<\delta<0.4$, solving the equations is difficult and extremely time consuming due to numerical problems. We were not able to characterize fully where and how the transition to the AS occurs.

Sparse connectivity: We consider here the case of a network of neurons which are partially and randomly connected. We assume that the synaptic weights, $J_{i j}$, are chosen as independent random variables, namely, $J_{i j}=g_{s} / M(i \neq j)$, with probability $M / N$ and $J_{i j}=0$ otherwise $\left(J_{i i}=0\right.$ for all $i$ ). On average a neuron receives input from $M$ other cells, so that the mean input into a cell is $g_{s} R$. Due to the randomness of the connectivity, the cells do not all exactly receive $M$ inputs. When $N<<M<<1$, cells receive input from approximately $M \pm \sqrt{M}$ other cells. The main effect of this is that in the asynchronous state different cells receive a constant feedback input which fluctuates spatially. Therefore, the neurons 
will have different firing rates and levels of adaptation. This can be captured in the large $\tau_{A}$ limit by describing the system in a coarse-grain time approximation where adaptation $A_{i}$ of neuron $i$ satisfies

$$
\tau_{A} \frac{d A_{i}}{d t}=-A_{i}+g_{A} r\left(I_{0}+g_{s} \bar{R} x_{i}-A_{i}\right)
$$

The function $r(I)$ is defined as above and $x_{i}=M_{i} / M$, where $M_{i}$ is the number of inputs cell $i$ receives. These differential equations have to be solved selfconsistently together with

$$
\bar{R}=\int d x \rho(x) r\left(I_{0}+g_{s} \bar{R} x-A_{i}\right),
$$

where $\rho$ is the distribution of $x_{i}=M_{i} / M$. In the large $M$ limit, this distribution will approach a narrowly peaked Gaussian with mean 1 , and standard deviation $1 / M$, and thus, the firing rates and adaptation will closely resemble that of a network where $x_{i}=1$ for all $i$. Therefore, as long as $M$ is sufficiently large, the network will show bursting behavior when a corresponding all-to-all coupled network does, irrespective of how small $M / N$ is. However, for sufficiently small $M$, $M<M_{c}$, bursting will be destroyed by the spatial fluctuations in connectivity. For instance, solving numerically Eqn. $(28,29)$ with $g_{A}=0.8$ and $g_{s}=0.675$, using the Gaussian approximation for $\rho(x)$, we find that $M_{c}<10$.

Noise: We consider a network in which the input $I_{i}(t)$ is given by

$$
I_{i}(t)=I_{0}+\eta_{i}(t)
$$

where the Gaussian white noise satisfies $<\eta_{i}(t)>=0$ and $<\eta_{i}(t) \eta_{j}\left(t^{\prime}\right)>=$ $\sigma^{2} \delta_{i j} \delta\left(t-t^{\prime}\right)$.

When $g_{A}=0$, in the AS, the neurons receive a total input with mean $I_{0}+g_{s} R\left(I_{0}\right)$ and uncorrelated fluctuations with standard deviation, $\sigma$. For such a network it can be shown (Tuckwell, 1988) that the rate $R$ satisfies

$$
R=\frac{\sigma}{\sqrt{2 \pi}}\left[\int_{x_{-}}^{x_{+}} d x e^{x^{2} / 2} H(x)\right]^{-1} .
$$

Here $x_{ \pm}=\left(I_{0} \pm g_{s} R\right) / \sigma$, and $H(x)$ is the complementary error-function $H(x)=$ $\int_{x}^{\infty} d x e^{-x^{2} / 2} / \sqrt{2 \pi}$. Equation (31) can be solved numerically for given $\sigma$. If $\sigma$ is not very large, the rate $R$ as a function of $I_{0}$ will have two stable branches. On 
one branch, which extends from $I_{0}=-\infty$ to $I_{0}=I_{1} \approx 1$, the rate $R$ is very low. We will denote this solution with $R_{-}\left(I_{0}\right)$. It corresponds to the quiescent state in the absence of noise. The second branch, on which the rate is substantial, extends from $I_{0}=I_{2}<I_{1}$ to $I_{0}=\infty$. This solution we will denote by $R_{+}\left(I_{0}\right)$ Below threshold, $I_{0}<1$, it corresponds to the self-sustained asynchronous state of the noiseless case. Stability analysis of the asynchronous state is non-trivial, but it can be shown that the first branch is always stable, while the second is also stable, provided that the noise is not to weak and the synaptic feedback is sufficiently strong.

Now consider the effect of adaptation. We assume that $\tau_{A}>>1$ and that $g_{A}>0$ satisfies:

$$
\frac{I_{0}-I_{2}}{R_{+}\left(I_{2}\right)}<g_{A}<\frac{I_{0}-I_{1}}{R_{-}\left(I_{1}\right)}
$$

As the noisy external input with a mean $I_{0}>1$ is turned on, the network will start to fire with rate $R_{+}\left(I_{0}\right)$ and the adaptation will grow from $A=0$ satisfying

$$
\tau_{A} \frac{d A}{d t}=-A+g_{A} R_{+}\left(I_{0}-A\right) .
$$

The adaptation grows until it reaches $A=I_{0}-I_{2}$. At this point, the rate drops to a much lower rate, $R_{-}\left(I_{2}\right)$, and the adaptation will decrease obeying

$$
\tau_{A} \frac{d A}{d t}=-A+g_{A} R_{-}\left(I_{0}-A\right)
$$

until the adaptation has reached $I_{0}-I_{1}$, at which point the rate rapidly jumps to $R_{+}\left(I_{1}\right)$. At this point, $A$ starts to grow again, satisfying Eqn. (33), etc.

It should be clear that the mechanism for bursting, both in the noisy and in the noiseless cases are similar. The only difference between the two cases is that in the noisy case, $g_{A}$ cannot be too large (see Eqn. 32). However, in practice this upper limit for $g_{A}$ is unrealistically large if $I_{0}$ is not very close to the threshold current.

\subsection{Realistic Adaptation Time Constants}

So far we have studied the dynamics of the network in the large $\tau_{A}$ limit. In fact, only for very large $\tau_{A}$, on the order of 1000 , the ratios $T_{B} / \tau_{A}$ and $T_{I} / \tau_{A}$ approach the large $\tau_{A}$ limit (result not shown). If we assume a membrane time constant, $\tau_{m}$, of $10 \mathrm{msec}$, this means that only for adaptation time constants on the order of 10 
seconds or larger does the theory predict $T_{B}$ and $T_{I}$ correctly. Clearly, realistic time constants for the adaptation are much smaller.

Thus, the theory does not give the right value for $T_{B}$ and $T_{I}$ when a realistic $\tau_{A}$ is used. Nevertheless, we can ask whether Eqns. (20) and (21) do at least give a qualitatively correct dependence of these two quantity on the different network parameters. In other words, we will check whether the theory allows us to understand how changing the network parameters changes the burst duration and the inter-burst interval.

Adaptation properties: In figure 9A, the time average burst duration and interburst interval are displayed as a function of $\tau_{A}$, for $g_{A}=0.6, g_{s}=0.85$ and $I_{0}=1.1$. Both quantities increase roughly linearly with $\tau_{A}$. However, as should be expected, the simulation results differ from the analytical estimate from the large $\tau_{A}$ limit theory, Eqn. (20) and (21).

Equation (21) implies that the interburst interval is independent of $g_{A}$, while Eqn. (20) shows that the burst duration diverges as $g_{A}$ approaches some critical value, $g_{A, c}$. For $g_{A}$ much larger than $g_{A, c}, T_{B}$ decreases as $1 / g_{A}$. In Fig. 9B, we show the dependence of $T_{B}$ and $T_{I}$ on $g_{A}$, in the large $\tau_{A}$ limit and from numerical simulations for $\tau_{A}=10$. Notice that these results have been obtained for moderately large values of $g_{A}$, and $g_{s}$, they are in excellent qualitative agreement with the analytical predictions. This is to be expected, since in our theoretical approach we do not assume weak coupling or adaptation. It should be noted noted however that there seems to be some extra structure, particularly in the interburst interval, which the large $\tau_{A}$ limit does not capture.

Synaptic coupling strength: According to our analysis of the bursting mechanism, there is a minimal value of $g_{s}, g_{s, c}$, for which $I_{c}=1$. This means that if $g_{s}<g_{s, c}$ neurons are not firing bursts. For $g_{s}$ just above $g_{s, c}, I_{c}$ is slightly larger than 1, so, according to Eqns. (20) and (21), $T_{B}$ and $T_{I}$ should be small. Increasing $g_{s}$ decreases $I_{c}$, and thus, according to Eqn. (21), $T_{I}$ will increase. This also tends to increase $T_{B}$. However, increasing $g_{s}$ also increases $R(I)$, which tends to decrease $T_{B}$. If $g_{s}$ is close to $g_{s, c}$ the increase in $I_{c}$ has the strongest effect and one expects that $T_{B}$ increases with $g_{s}$. By contrast, when $g_{s}$ approaches its maximum value, $g_{s}=1$ (in the large $\tau_{A}$ limit), $I_{c}$ approaches slowly its minimum value, $I_{c}=0.5$. But here, the rate, $R(I)$, increases very rapidly with $g_{s}(R(I) \rightarrow \infty$ for $\left.g_{s} \rightarrow 1\right)$. Thus for sufficiently large $g_{s}$, the increase in the firing rate, $R$, is the dominant factor. Therefore, for large $g_{s}, T_{B}$ decreases with $g_{s}$. As $g_{s}$ approaches $1, T_{B}$ approaches 0 .

As is also shown in Fig. 9C, for $\tau_{A}=100$, the prediction of the theory is 


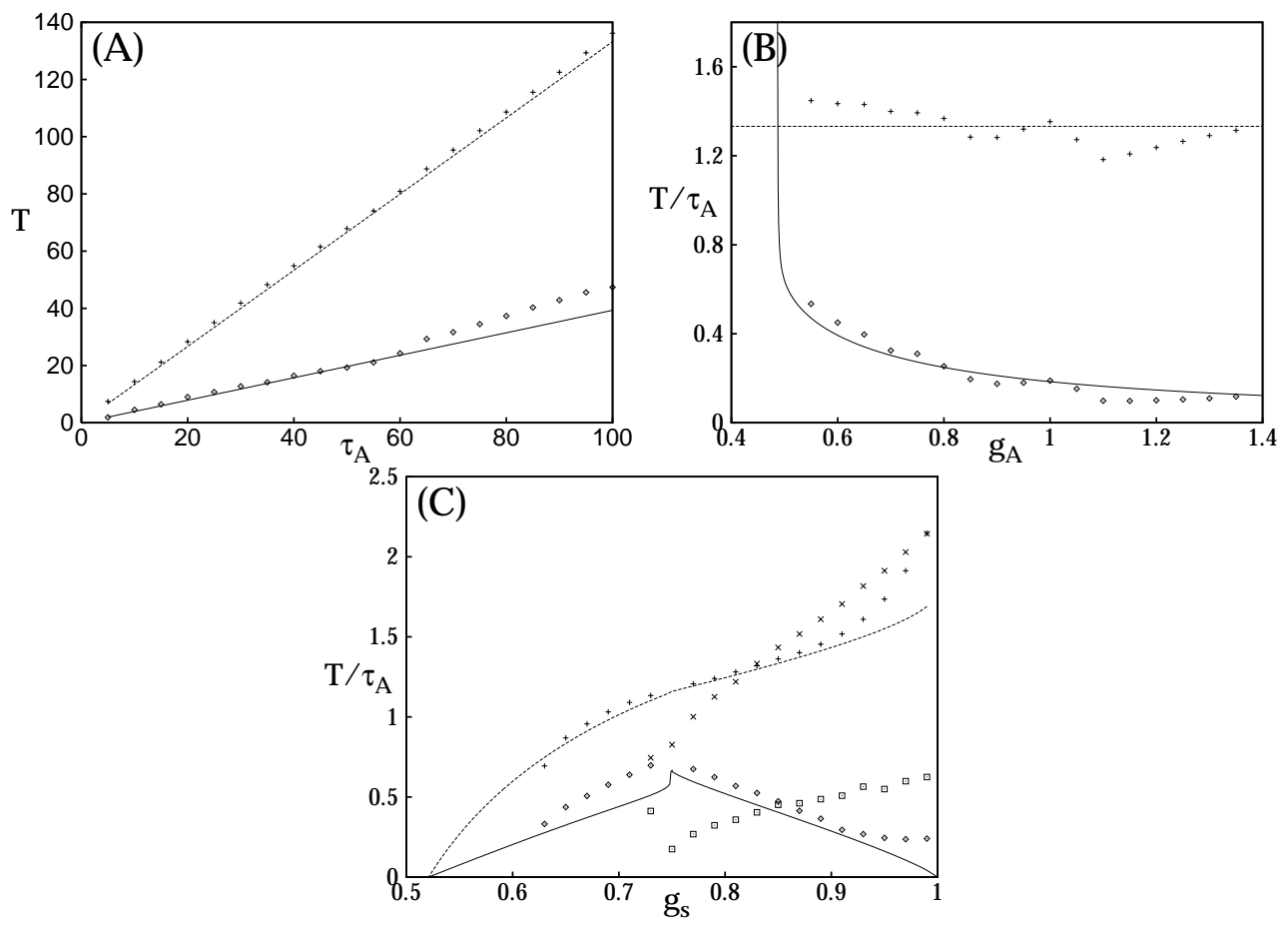

Figure 9: Results from numerical simulations of a network with $N=200$ excitatory neurons. Synaptic rise time: $\tau_{1}=0.1$; Synaptic decay time: $\tau_{2}=0.3$. A: $T_{B}$ and $T_{I}$ (in units of $\tau_{m}$ ) versus $\tau_{A}$. Solid line: theoretical value of $T_{B}$ from large $\tau_{A}$ limit, dashed line: theoretical value of $T_{I}$ from large $\tau_{A}$ limit, diamonds: $T_{B}$ in simulation, pluses: $T_{I}$ in simulation. Parameters: $I_{0}=1.1, g_{s}=0.85, g_{A}=0.6 . \mathbf{B}: T_{B} / \tau_{A}$ and $T_{I} / \tau_{A}$ versus $g_{A}$. Solid line: theoretical value of $T_{B} / \tau_{A}$, dashed line: theoretical value of $T_{I} / \tau_{A}$, diamonds: $T_{B} / \tau_{A}$ in simulation with $\tau_{A}=10$, pluses: $T_{I} / \tau_{A}$ in simulation with $\tau_{A}=10$. Parameters: $I_{0}=1.1, g_{s}=0.85$. C: $T_{B} / \tau_{A}$ and $T_{I} / \tau_{A}$ versus $g_{s}$. Solid line: theoretical value of $T_{B} / \tau_{A}$, dashed line: theoretical value of $T_{I} / \tau_{A}$, diamonds: $T_{B} / \tau_{A}$ in simulation with $\tau_{A}=10$, pluses: $T_{I} / \tau_{A}$ in simulation with $\tau_{A}=10$, squares: $T_{B} / \tau_{A}$ in simulation with $\tau_{A}=100$, crosses: $T_{I} / \tau_{A}$ in simulation with $\tau_{A}=100$. Parameters: $I_{0}=1.1, g_{A}=0.6$. 
confirmed. However, if $\tau_{A}$ is too small, e.g., $\tau_{A}=10$, our numerical simulations show a very different behavior for $T_{B}$ as a function of $g_{s}$. Indeed, for this value of $\tau_{A}$, the burst duration, $T_{B}$, does not decrease as $g_{s}$ approaches 1 , unlike in the large $\tau_{A}$ limit. The firing rate during the burst also stays finite in this limit, contrary to what one expects from the large $\tau_{A}$ limit. This difference is due to the fact that in the theoretical treatment of the large $\tau_{A}$ limit, we assumed that the time needed to settle in the AS was negligible, not only compared to $\tau_{A}$, but also compared to $T_{B}$ and $T_{I}$. When the excitatory coupling approaches $g_{s}=1$, this is no longer valid, since in this limit $T_{B}$ becomes small. Thus, the discrepancy between theory and simulation for $\tau_{A}$ which is too small is to be expected.

Synaptic coupling strength: We have also checked, using numerical simulations, that for realistic adaptation time constant, the bursting state is robust to heterogeneities and sparseness using numerical simulations. Our results were in agreement with the conclusions of the large $\tau_{A}$ theory. For instance, for the parameters of Section 4.4, and $\tau_{A}=10$, the network settles in a bursting state for $\delta$ as large as 0.3 .

\section{Two Populations}

Up to this point, we have analyzed the collective bursting state which emerges in a network of excitatory neurons with spike adaptation, when the synaptic feedback is sufficiently strong. Based on numerical simulations, we have also shown that these states are robust to noise, heterogeneities of intrinsic neuronal properties, and to sparseness of the connectivity. In this section, we study what happens to this bursting state when a second population, consisting of inhibitory neurons without spike adaptation, interacts with this excitatory population.

Several experimental and numerical studies have shown (Silva et al., 1991, Flint and Connors, 1996) that if, in cortical slices, the feedback from inhibitory neurons is blocked, the activity of the circuit can change from tonically active to synchronized burst activity. An interesting question is whether or not our model can account for this change in activity. To investigate this, we consider a network that consists of two populations of neurons, one excitatory and one inhibitory.

The large $\tau_{A}$ limit: As in the one population network, the system becomes much simpler to analyze in the limit $\tau_{A} \rightarrow \infty$. In this limit, the system will show activity with spike to spike synchrony. However, if the properties of the neurons are sufficiently inhomogeneous, the connectivity is sparse, and $g_{I I}$ is sufficiently 
small, then the network will evolve to an asynchronous state. In this case one can, similarly to the one population model, reduce the system to a system described by

$$
\tau_{A} \frac{d A}{d t}=-A+g_{A} R_{E}(A)
$$

Where the rate, $R_{E}(A)$, has to be determined self-consistently from

$$
\begin{aligned}
R_{E} & =f\left(I_{0}^{E}+G_{E E} R_{E}+G_{I I} R_{I}-A\right) \\
R_{I} & =f\left(I_{0}^{I}+G_{I E} R_{E}+G_{I I} R_{I}\right) / \tau_{I},
\end{aligned}
$$

where $f(I)=[\log (I /(I-1))]^{-1}$. Since $f(I)$ is a non-trivial function of $I$, there is no explicit solution to this set of equations. Nevertheless, if $I_{0}^{I}>1$, the firing rate of the inhibitory population, $R_{I}$, can be approximated by $R_{I}=$ $\left(I_{0}^{I}+G_{I E} R_{E}-1 / 2\right) /\left(\tau_{I}-G_{I I}\right)$. In this case, $R_{E}$ satisfies

$$
R_{E}=f\left(I_{0}^{E}+G_{E I} \frac{I_{0}^{I}-1 / 2}{\left(\tau_{I}-G_{I I}\right)}-A+\left[G_{E E}+\frac{G_{E I} G_{I E}}{\left(\tau_{I}-G_{I I}\right)}\right] R_{E}\right) .
$$

This shows that the net effect of adding the inhibitory population is to reduce the external input into the excitatory neurons from $I_{0}=I_{0}^{E}$ to $I_{0}^{e f f}=I_{0}^{E}+$ $G_{E I}\left(I_{0}^{I}-1 / 2\right) /\left(\tau_{I}-G_{I I}\right)$, and decreasing the synaptic strength of the excitatory to excitatory connections from $g_{s}=G_{E E}$ to $g_{s}^{\text {eff }}=G_{E E}+G_{E I} G_{I E} /\left(\tau_{I}-G_{I I}\right)$. (Recall that $G_{E I}$ is negative.)

Now consider the situation where, in the single population, $g_{s}$ and $I_{0}$ are sufficiently large, so that this single population would evolve to the bursting state. Then, the inhibitory network feedback can reduce the effective external input, $I_{0}^{e f f}$, and coupling, $g_{s}^{e f f}$, sufficiently, to stabilize the tonically firing state, provided that $G_{E I}$ and $G_{I E}$ are sufficiently large. Thus, in such a network the neurons fire tonically. However, chemically blocking the gabaergic synapses, i.e., setting $G_{E I}$ to zero, will result in synchronized burst activity of the excitatory population.

\section{Conductance-based Networks with Adaptation}

In this section, we show that the results we have established for IF network models can be generalized in the framework of conductance-based neuronal networks. These models take into account in a more plausible way the biophysics underlying action potential firing but they do not yield to analytical study, even in the limit 
of slow adaptation. Therefore, our study of these models relies only on numerical simulations.

The neurons obey the following dynamical equations:

$$
\begin{aligned}
C \frac{d V_{i}(t)}{d t} & =g_{L}\left(E_{L}-V_{i}(t)\right)-I_{i}^{\text {active }}(t) \\
& +I_{i}^{\text {app }}(t)+I_{i}^{\text {syn }}(t), \quad(i=1, \ldots, N)
\end{aligned}
$$

where $V_{i}(t)$ is the membrane potential of the $i$-th cell at time $t, C$ is its capacitance, $g_{L}$ is the leak conductance and $E_{L}$ is the reversal potential of the leak current. The leak conductance, $g_{L}$, represents the purely passive contribution to the cell's input conductance, and is independent of $V_{i}$ and $t$. In addition to the leak current, the cell has active ionic currents with Hodgkin-Huxley type kinetics (Tuckwell 1988), the total sum of which is denoted as $I_{i}^{\text {active }}(t)$ in Eqn. (39). They lead to a repetitive firing of action potentials if the cell is sufficiently depolarized. An externally injected current is denoted as $I^{a p p}$. The synaptic coupling between the cells gives rise to a synaptic current, $I^{s y n}$, which is modeled as

$$
I_{i}^{s y n}(t)=\sum_{j=1}^{N} g_{i j}(t)\left(E_{j}-V_{i}(t)\right)
$$

where $g_{i j}(t)$ is the synaptic conductance triggered by the action potentials of the presynaptic $j$-th cell, and $E_{j}$ is the reversal potential of the synapses made by neuron $j$.

The synaptic conductance is assumed to consist of a linear sum of contributions from each of the presynaptic action potentials. The detailed dynamical equations are given in Appendix B.

The neurons in the network receive an external input from a set of neurons which fire spikes at random with a Poisson distribution. The effect of this external input is represented in Eqn. (39) by the current $I_{i}^{\text {stim }}(t)$. This current depend on the average rate of the input neurons, on the peak conductance and the time constants of their synapses as explained in Appendix B.

One excitatory population: We first consider the limit where all of the inhibitory interactions are blocked. If the excitation is weak, the network settles into an asynchronous state. This is shown in Fig. 10A. Increasing the synaptic strength, leads to synchronized burst states in a very similar way to what we have found for the IF network. The spikes within the bursts are not synchronized, see Fig. 10B. 

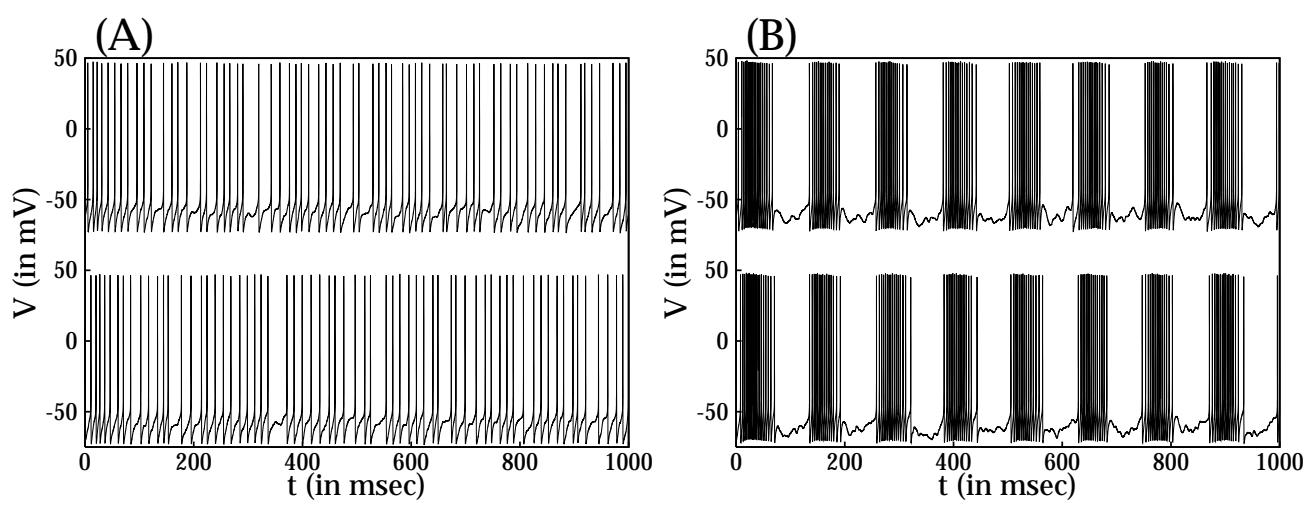

Figure 10: A: Activity of two cells in a population of weakly coupled excitatory conductance based neurons. The neurons fire tonically in an asynchronous state. Coupling strength: $G_{E E}=$ $0.2 \mathrm{mS} / \mathrm{cm}^{2}$. The network consists of $N=200$ all-to-all coupled neurons. The external input has an average firing rate, $f_{0}=1300 \mathrm{~Hz}$, and a conductance, $G_{0}=0.03 \mathrm{mS} / \mathrm{cm}^{2}$. The parameters of the single neuron dynamics and of the synapses are given in Appendix B. B: Activity of two neurons in a strongly coupled population of excitatory conductance based neurons. Coupling strength: $G_{E E}=0.6 \mathrm{mS} / \mathrm{cm}^{2}$. Same parameters as in A. The neurons fire in synchronized bursts without spike to spike synchrony.

We have also studied the dependence of the interburst duration and of the intraburst duration as a function of $\tau_{A}$ and $g_{A}$. The results are shown in Fig. 11 and are qualitatively similar to what we have found for the IF network (compare with Figs. 9A, 9B). Finally, we have studied the average synaptic contact per neuron required for the collective bursting state to emerge. This number is on the order of 5 to 10 , as for the I\& F network.

Two population network: We now consider the two population conductance based neuronal network. For the sake of simplicity, we assume that the dynamics of the inhibitory population involve the same currents as the excitatory one, except that the adaptation current is suppressed. The maximal conductances of the synapses that neurons in population $\beta$ are making on neurons in population $\alpha$ $(\alpha, \beta=E, I)$ is denoted by: $G_{\alpha \beta}$. The connectivity of the neurons inside each population and between them are sparse: the number of synaptic inputs fluctuates from neuron to neuron with an average of $M=100$ synaptic inputs. In the following, we fix all the parameters of the network and we discuss the dependence of the network state on the inhibitory conductances $G_{I I}$ and $G_{E I}$. We have checked the behavior of the network for other parameter sets and we have found that these results are qualitatively generic for a broad range of parameters, provided that the 

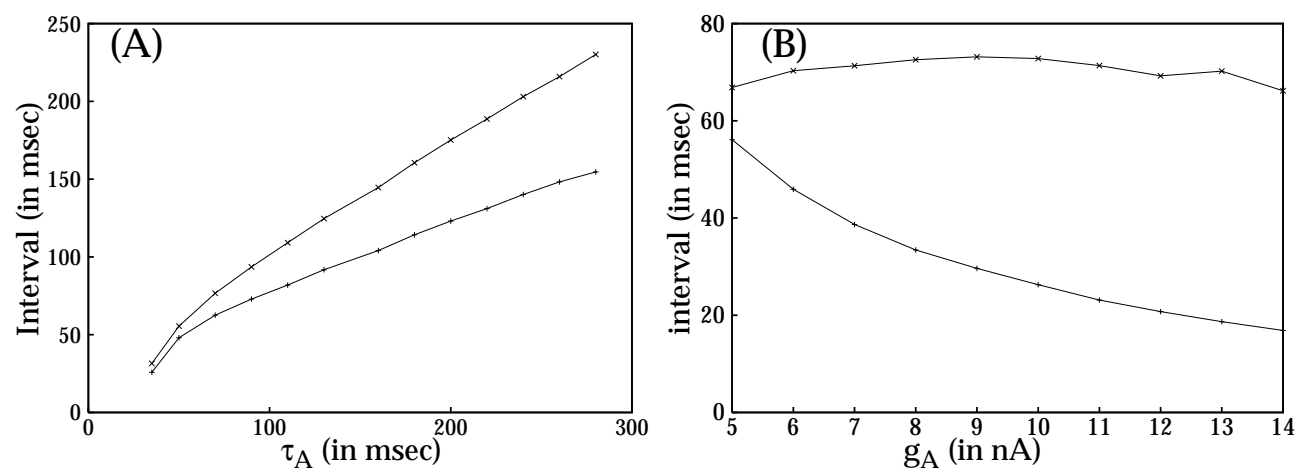

Figure 11: A: $T_{B}$ and $T_{I}$ versus $\tau_{A}$. Pluses: $T_{B}$, Crosses: $T_{I} . G_{E E}=0.6 \mathrm{mS} / \mathrm{cm}^{2} ; f_{0}=$ $2600 \mathrm{~Hz} ; G_{0}=0.015 \mathrm{mS} / \mathrm{cm}^{2} ; g_{A}=5 \mathrm{mS} / \mathrm{cm}^{2}$. Other parameters as in Appendix B. B: $T_{B}$ and $T_{I}$ versus $g_{A}$. Pluses: $T_{B}$, Crosses: $T_{I} . \tau_{A}=60 \mathrm{msec}$; other parameters as in A.

adaptation strength is sufficient.

Fig. 12 displays the coherence of the network as a function of $G_{E I}$ and $G_{I I}$. When the inhibition is sufficiently weak, with $G_{E I}$ and $G_{I I}$ both small, the system settles into a highly synchronous bursting state. By increasing $G_{E I}$, keeping $G_{I I}$ fixed and not too large, the network remains in a bursting state, but the level of coherence of the network decreases, while the length of the bursts decreases as well as the average firing rate of the excitatory population. At the same time, the firing rate of the inhibitory population increases. Eventually, if $G_{E I}$ is above a critical value the network becomes asynchronous and the neurons are not firing bursts of spikes any more. This critical value increases with $G_{I I}$. Note also that for fixed $G_{E I}$, the degree of synchrony decreases with $G_{I I}$. This is due to the fact, that decreasing the inhibition between the inhibitory neurons, decreases the effective gain of the network (see Eqn. (37)).

\section{Conclusion}

Our study deals with stimulated and supra-threshold strongly interacting neurons. The case of subthreshold excitatory neurons with adaptation has recently been addressed by Golomb and Amitai (1996). Synchrony of weakly interacting suprathreshold excitatory neurons with spike adaptation has been investigated by Crook et al (1996). The mechanisms for synchrony they have studied differ essentially from the one we have investigated here which involves strong excitatory feedback. A mechanism involving a cooperation of strong excitation and with slow 


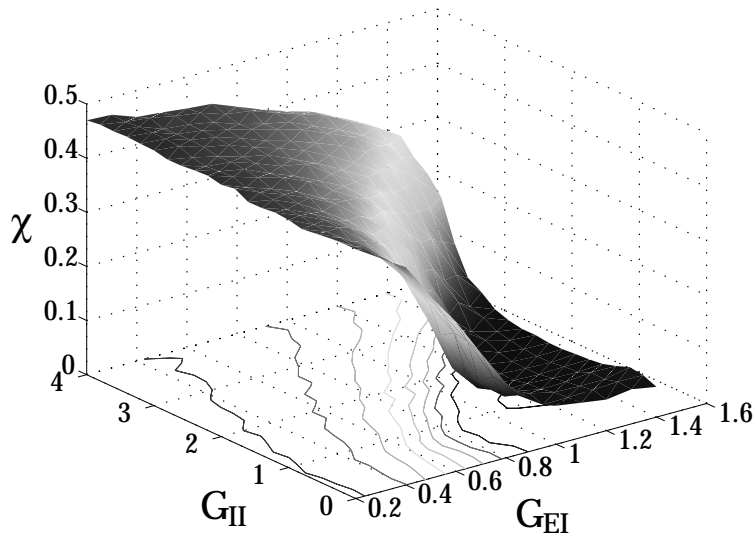

Figure 12: Coherence $\chi$ against $G_{I I}$ and $G_{E I}$. The network consists of $N_{E}=800$ excitatory and $N_{I}=800$ inhibitory sparsely connected. Each neuron receives in average 89 inputs excitatory and 89 inputs inhibitory. $G_{E E}=0.8 \mathrm{mS} / \mathrm{cm}^{2}, G_{I E}=0.8 \mathrm{mS} / \mathrm{cm}^{2}, g_{A}=8 \mathrm{mS} / \mathrm{cm}^{2}, \tau_{A}=$ $60 \mathrm{msec}$. The rate of the external input on the excitatory (resp. inhibitory) population is $336 \mathrm{~Hz}$ (resp. $140 \mathrm{~Hz}$ ). The conductance of the external input is $0.105 \mathrm{mS} / \mathrm{cm}^{2}$ on both populations. All other parameters are as indicated in Appendix B.

ionic current has been investigated recently by Butera et al. for the generation of respiratory rhythm (Butera et al., 1999). Latham et al. consider a network of intrinsically active neurons with spike adaptation which exhibits a bursting mechanism similar to ours (Latham et al. 1999).

We have examined a simple scenario that can lead to rhythmic and stable collective patterns of neural activity. We have shown that states characterized by synchronization and slow bursting emerge naturally from a cooperation between excitatory feedback and firing adaptation. In these states, neurons fire bursts which are synchronized, but the spikes within the bursts are not. The bursting this system exhibits is a network effect, since the neurons cannot fire periodic bursts on their own. It relies on the fact that an excitatory network can display a region of bistability and on a slow process which drags the neurons back and forth in and out of this bistable region. This mechanism is very similar to those that have been proposed for single neuron bursting (Rinzel and Ermentrout, 1998).

This synchronized bursting is a general phenomenon. As we have shown, it occurs for the I\&F network as well as for more biophysically plausible conductancebased neuronal models. In both classes of models, the synchronized bursting states have similar properties. It is also extremely robust to heterogeneity, sparseness of the connectivity, and noise. This is in contrast to mechanisms of spike synchrony in excitatory or inhibitory networks (White et al., 1998, Neltner et al., 
1999, Golomb and Hansel, 1999).

We have dissected analytically the mechanism of this phenomenon in a network of one population of excitatory I\&F neurons, assuming slow adaptation $\left(\tau_{A} \rightarrow \infty\right)$. In this limit, a simple equation can be written to relate the time evolution of the adaptation current of the neurons and their firing rate. Another assumption of our analysis is that without adaptation the network settles into either an asynchronous state, when this state is stable, or into a quiescent state when the former is unstable. Under this assumption the dynamics of the excitatory networks can be reduced to a "rate model" in which the dynamics of the neurons are characterized by a rate variable which corresponds to the adaptation current. This is in contrast to other types of rate models in which the dynamical quantity corresponds to a population activity (Wilson and Cowan, 1972, Ginzburg and Sompolinsky, 1994) or to synaptic conductances (Ermentrout, 1994, Shriki et al, 1999). Another difference with rate models introduced previously, is that in our case, rate equations have to be supplemented with the stability condition of the asynchronous state of the full spiking model. This approach should be contrasted to the one taken in Latham et al. 1999, where the population activities are described by Wilson and Cowan equations. This is equivalent to our approach under the assumption that if the AS is always stable if it exists. Also note that in order to derive Eqns.(18,17), we have also assumed that the synaptic time constant is fast. If one assumes that the synaptic time constants are large, of the same order of magnitude than the adaptation time constant, one finds more complicated rate dynamics equations. They involve two coupled "rate variables". One corresponds to the adaptation current and the other to the synaptic current.

Relying on numerical simulations, we have investigated the network dynamics for finite values of $\tau_{A}$. Our results show that quantitative agreement between simulations and our large $\tau_{A}$ theory requires very large and unrealistic values of $\tau_{A}$. However, for reasonable values of $\tau_{A}$, we have found good qualitative agreement. One of the discrepancies between the theory and the simulations is due to the fact that, even in the large $\tau_{A}$ limit, there is always a small but finite fraction of the burst in which the spikes are synchronized across the network. Adding a small amount of heterogeneities changes the picture. Indeed, in this limit, the fraction of the burst during which the spikes remain synchronized during a burst goes to 0 when $\tau_{A}$ diverges. Therefore, there is a cross-over between the limit of no heterogeneities and the limit $\tau_{A} \rightarrow \infty$. However, an extremely large $\tau_{A}$ is required to see this cross-over in numerical simulations.

We have also shown how the effect of adding an inhibitory population can be understood in the large $\tau_{A}$ limit. We have seen that, recurrent inhibition reduces 
the effective excitatory feedback and the external current, and that this reduction depends on the strength of the inhibition between the inhibitory neurons. This can be sufficient to settle the network in an asynchronously firing state, even if the network without inhibition would evolve to the synchronized bursting state. This explains how chemically blocking the inhibitory feedback can change the activity pattern of the network from asynchronous tonically firing to synchronized bursts (Silva et al., 1991, Connors et al., 1996). The same effects can be observed in networks with realistic adaptation time constants. The fact that inhibition destroys the bursting state has to be opposed to the synchronizing effect of inhibition in spike to spike synchrony (Hansel et al. 1993, Van Vreeswijk et al. 1994, Hansel et al. 1995).

The pattern of firing of the neurons in the synchronous bursting state is reminiscent of epileptic population bursting. A mechanism proposed to explain the emergence of population bursting in the hippocampus involves excitatory coupling between neurons which are able to fire bursts on their own (Traub, 1992, Pinsky and Rinzel, 1994). Our study shows that very similar collective states can be achieved with non-bursting neurons. Since adaptation currents display a wide spectrum of time constants ranging from tens to hundreds of msec, this leads very naturally to rhythmic activity over a wide range of frequencies.

In this paper, we have focused on networks with random connectivity, as observed, e.g., in the hippocampus. Although it is still a matter of debate, this type of architecture is probably also relevant for modeling the dynamics of motor cortical areas. The somatosensory and the visual cortex are organized differently. The connectivity pattern in these area is highly correlated with the functional properties of the neurons. In the primary visual cortex, for instance, the probability of connection between two neurons decreases with the difference of their preferred orientation. In networks with this type of architecture, collective bursting can also occur. However, if the inhibition in the network is sufficiently strong, the collective and synchronous bursting state can be destabilized and replaced by a traveling pulse state, as has recently been shown (Hansel and Sompolinsky, 1998).

It is worth noting that the mechanism described here is not the only one can induce burst activity in a network that consists of neurons that fire tonically if they are isolated and injected with a constant current. In a recent paper (O'Donovan et al., 1998) it was shown that excitatory networks of neurons with synaptic adaptation can also bifurcate to a bursting state. These bursts can have a similar time scale as the bursts in our network. It would be interesting to see how an interplay of these two mechanisms affect the network activity. 


\section{Acknowledgments}

We are thankful to D. Golomb, G. Mato, and P. Dayan for careful reading of the manuscript. D.H. acknowledges hospitality of the Center for Neural Computation and the Racah Institute of Physics of the Hebrew University. This research was partially supported by the PICS 236 (PIR Cognisciences and M.A.E): “ Dynamique neuronale et codage de l'information: Aspects physiques et biologiques" to D.H.

\section{Appendix A: Bursts properties in weakly hetero- geneous network}

In this appendix, we study how a weak level of heterogeneities in the external input affects the synchronized bursting state of the excitatory I\&F network. The equation for the membrane potential of neuron $i$ is:

$$
\frac{d}{d t} V_{i}=-V_{i}-A_{i}+I_{i}+g_{s} E
$$

where $I_{i}=I_{0}+\delta I_{i}$, with $N^{-1} \sum_{i} \delta I_{i}=0$. If the inhomogeneities $\delta I_{i}$ are small, $\sigma(I) \equiv N^{-1} \sum_{i}\left(\delta I_{i}\right)^{2} \ll 1$, we can expand around the solution with homogeneous input. It can be shown that $I_{c}$ will shift by a small amount, $\delta I_{c}$ that is of the order of $\sigma(I)$. We will assume that $\sigma(I)$ is small enough, so that this shift in $I_{c}$ can be ignored.

We will now show that under these conditions the burst duration and interburst interval satisfy Eqns. (20) and (21) to leading order.

We assume that the network has reached a state where the average rate, $R$, varies periodically, and that $R>0$ for $0<t<T_{B}$, while $R=0$ for $T_{B}<$ $t<T_{B}+T_{I}$. Thus, we have to show in this case that level of synchronization is small enough at the beginning of the burst, so that the time it takes to reach the asynchronous state is negligible compared to $T_{B}$ in the large $\tau_{A}$ limit. Writing $R_{i}(t)=R(t)+\delta R_{i}(t)$ and $A_{i}(t)=A(t)+\delta A_{i}(t)$, we find that when the network fires asynchronously,

$$
\frac{1}{R+\delta R_{i}}=\int_{0}^{1} \frac{d V}{I_{0}+g_{s} R-A-V+\delta I_{i}-\delta A_{i}} .
$$

Or, to leading order,

$$
\delta R_{i}(t)=\left(\delta I_{i}-\delta A_{i}(t)\right) G(t)
$$


with $G(t)=R^{2}(t) \int_{0}^{1} d V /\left(I_{0}+g_{s} R(t)-A(t)-V\right)^{2}$. Here, $R(t)$ and $A(t)$ are the solutions for the rate and adaptation currents derived in section 5. Thus, $\delta A_{i}$ satisfies

$$
\tau_{A} \frac{d \delta A_{i}}{d t}= \begin{cases}-\delta A_{i}+g_{A}\left(\delta I_{i}-\delta A_{i}\right) G(t) & \text { for } 0<t<T_{B} \\ -\delta A_{i} & \text { for } T_{B}<t<T_{B}+T_{I},\end{cases}
$$

and

$$
\delta A_{i}(0)=\delta A_{i}\left(T_{B}+T_{I}\right)
$$

These equations imply that $\delta A_{i}$ has a linear dependence on $\delta I_{i}, \delta A_{i}(t)=$ $-a(t) \delta I_{i}$, where $a$ is a periodic function with period $T_{B}+T_{I}$. The precise shape of $a(t)$ depends on the network parameters. However, (because of the nonhomogeneous term present in the RHS of Eqn. (A.4)) for all parameter choices $0<a(t)<1$ for all $t$. Since $a\left(T_{B}\right)<1, a\left(T_{B}+T_{I}\right)$, thus, $a(0)$ can at most have the value $a_{\max }=e^{-T_{I} / \tau_{A}}$.

At $t=T_{B}^{+}$, just after the termination of the burst, the standard deviation of the membrane potentials, $\sigma_{V}$, is of the order 1 . In the interburst interval, $\sigma_{V}$ satisfies

$$
\frac{d}{d t} \sigma_{V}^{2}=-2 \sigma_{V}^{2}+(1-a(t))<V \delta I>
$$

where $<V \delta I>=N^{-1} \sum_{i} V_{i} \delta I_{i}$ obeys

$$
\frac{d}{d t}<V \delta I>=-<V \delta I>+(1-a(t)) \sigma_{I}^{2}
$$

Thus, if we neglect the term with $d a / d t$ which is of order $1 / \tau_{A}$, we find for $\sigma_{V}^{2}$

$$
\left(\frac{d^{2}}{d t^{2}}+3 \frac{d}{d t}+2\right) \sigma_{V}^{2}=(1-a(t))^{2} \sigma_{I}^{2} .
$$

Therefore, just before the next burst begins, the standard deviation in the membrane potential will at least be equal to

$$
\sigma_{V}^{2}\left(T_{B}+T_{I}\right) \geq\left(1-e^{-T_{I} / \tau_{A}}\right)^{2} \sigma_{I}^{2}
$$

So, it will take a time of order $-\log \left(\left(1-e^{-T_{I} / \tau_{A}}\right)^{2} \sigma_{I}^{2}\right) / \lambda$ before the asynchronous state is reached. So, as long as $-\log \left(\sigma_{I}^{2}\right)$ is large compared to $\tau_{A}$, it will take a negligible fraction of the burst duration before the cells fire asynchronously. 
Consequently, Eqns. (20) and (21) will predict $T_{B}$ and $T_{I}$ correctly in the large $\tau_{A}$ limit.

Thus, independent of the length of the burst, the standard deviation in the membrane potential is at least $\sigma_{I}$ at the start of the burst. This means that it will take, at most, a time of order $-\log \left(\sigma_{I}\right) / \lambda$ after the commencement of the burst until $\sigma_{V}$ is of order 1 . So, in the large $\tau_{A}$ limit it takes an arbitrarily small fraction of the burst duration until the network is again in the asynchronous state.

It should be noted that in the presence of inhomogeneity in the input, the cells do not all fire at the same rate, and, therefore, the adaptation currents, $A_{i}$, will not go to the same value. This inhomogeneity will also affect $I_{c}$. However, if $\sigma_{I}$ is small, this will only have a small effect on $T_{B}$ and $T_{I}$. So, if a small inhomogeneity is added to the input, Eqns. (20) and (21) will give a good approximation of $T_{B}$ and $T_{I}$ in the large $\tau_{A}$ limit.

\section{Appendix B: The conductance based model}

In this appendix, we give the details of the equations of the conductance based model we have used in Section 6.

The membrane potentials of the neurons follow the equation:

$$
C \frac{d V}{d t}=-I_{\mathrm{L}}-I_{\mathrm{Na}}-I_{\mathrm{K}}-I_{\mathrm{KA}}-I_{\mathrm{adapt}}+I_{\mathrm{syn}}(t)+I_{\mathrm{ext}}(t)
$$

where $I_{\text {syn }}$ denotes the synaptic current generated within the network and $I_{\text {ext }}$ stands for synaptic currents from sources outside the network.

The leak current is $I_{\mathrm{L}}=g_{\mathrm{L}}\left(V-E_{\mathrm{L}}\right)$. The sodium and the delayed rectifier currents are described in a standard way: $I_{\mathrm{Na}}=g_{\mathrm{Na}} m_{\infty}^{3} h\left(V-E_{\mathrm{Na}}\right)$, for the sodium current and $I_{\mathrm{K}}=g_{\mathrm{K}} n^{4}\left(V-E_{\mathrm{K}}\right)$, for the delayed rectifier current. The gating variables, $x=h, n$ satisfy the relaxation equations: $d x / d t=\left(x_{\infty}-x\right) / \tau_{\mathrm{x}}$. The functions $x_{\infty},(x=h, n, m)$, and $\tau_{\mathrm{x}}$ are: $x_{\infty}=\alpha_{\mathrm{x}} /\left(\alpha_{\mathrm{x}}+\beta_{\mathrm{x}}\right)$, and $\tau_{\mathrm{x}}=\phi /\left(\alpha_{x}+\beta_{x}\right)$ where $\alpha_{\mathrm{m}}=-0.1(V+30) /(\exp (-0.1(V+30))-1), \beta_{\mathrm{m}}=4 \exp (-(V+55) / 18)$, $\alpha_{\mathrm{h}}=0.07 \exp (-(V+44) / 20), \beta_{\mathrm{h}}=1 /(\exp (-0.1(V+14))+1), \alpha_{\mathrm{n}}=$ $-0.01(V+34) /(\exp (-0.1(V+34))-1)$ and $\beta_{\mathrm{n}}=0.125 \exp (-(V+44) / 80)$. We have taken: $\phi=10$. The model incorporates a potassium $A$-current: $I_{\mathrm{KA}}=g_{K A} a_{\infty}^{3} b\left(V-E_{\mathrm{K}}\right)$ with $a_{\infty}=1 /(\exp (-(V+50) / 20)+1)$. The function $b(t)$ is given by: $d b / d t=\left(b_{\infty}-b\right) / \tau_{\mathrm{KA}}$ with: $b_{\infty}=1 /(\exp ((V+80) / 6)+1)$. For the sake of simplicity, the time constant, $\tau_{\mathrm{KA}}$ is voltage independent. The adaptation current, denoted by $I_{\text {adapt }}$, is modeled as: $I_{\text {adapt }}=g_{\mathrm{A}} A(t)\left(V-E_{\mathrm{K}}\right)$ 
where the function $A(t)$ satisfies:

$$
\frac{d A}{d t}=\frac{A_{\infty}(V)-A}{\tau_{\mathrm{A}}}
$$

where $A_{\infty}(V)=1 /(\exp (-0.7(V+30))+1$.$) For the sake of simplicity, the$ adaptation time constant $\tau_{\mathrm{A}}$ is independent of the voltage.

The other parameters of the model are: $C=1 \mu \mathrm{F} / \mathrm{cm}^{2}, g_{\mathrm{Na}}=100 \mathrm{mS} / \mathrm{cm}^{2}$, $g_{\mathrm{K}}=40 \mathrm{mS} / \mathrm{cm}^{2}$. Unless otherwise specified, $g_{\mathrm{L}}=0.05 \mathrm{mS} / \mathrm{cm}^{2}, g_{\mathrm{KA}}=$ $20 \mathrm{mS} / \mathrm{cm}^{2}$ and $\tau_{K A}=20 \mathrm{msec}$. The reversal potentials of the ionic and synaptic currents are: $E_{\mathrm{Na}}=55 \mathrm{mV}, E_{\mathrm{K}}=-80 \mathrm{mV}, E_{\mathrm{L}}=-65 \mathrm{mV}, E_{\mathrm{E}}=0 \mathrm{mV}$ and $E_{\mathrm{I}}=-80 m V$.

The synaptic inputs from inside the network are modeled as conductance changes. The synaptic current flow into a postsynaptic cell at time $t$, induced by a single presynaptic spike at time $t_{0}$, is

$$
I_{\text {syn }}(t)=G_{\text {syn }} f\left(t-t_{0}\right)\left(E_{\text {syn }}-V(t)\right)
$$

where $V(t)$ is the membrane potential of the postsynaptic neuron at time $t, E_{\mathrm{syn}}$, the reversal potential of the synapse, and $G_{\text {syn }}$, its strength. The function $f(t)$ is given by:

$$
f(t)=\frac{1}{\tau_{1}-\tau_{2}}\left(e^{-\frac{t}{\tau_{1}}}-e^{-\frac{t}{\tau_{2}}}\right) \Theta(t)
$$

$\left(\Theta(t)\right.$ is the Heaviside function). The larger of the time constants, $\tau_{1}$ and $\tau_{2}$, characterizes the rate of exponential decay of the synaptic potentials, while their time to peak is equal to $t_{\text {peak }}=\left(\tau_{1} \tau_{2} /\left(\tau_{1}-\tau_{2}\right)\right) \ln \left(\tau_{1} / \tau_{2}\right)$. The normalization adopted here ensures that the time integral of $f(t)$ is always unity. For multiple events, $I_{\text {syn }}(t)$ becomes the sum of the total contributions at time $t$ of all spikes generated by the presynaptic cells in the past.

The external synaptic inputs which are excitatory, are described in a similar way as for the internal synaptic inputs. The spike times, $t_{0}$, are random and taken from a Poisson process with a fixed uniform rate, $f_{0}$. The external synaptic inputs to different cells are uncorrelated.

The synaptic time constants are: $\tau_{1}=1 \mathrm{msec}, \tau_{2}=3 \mathrm{msec}$ for the excitation in the network; $\tau_{1}=1 \mathrm{msec}, \tau_{2}=6 \mathrm{msec}$ for the inhibition. The synapses from outside the network are have an instantaneous rise. They decay with a time constant $\tau_{2}=3$ msec. 


\section{Appendix C: Measure of synchrony in large neu- ronal networks}

To measure the degree of synchrony in the network, we follow the method proposed and developed in (Hansel and Sompolinsky, 1992, Golomb and Rinzel, 1993, Ginzburg and Sompolinsky, 1994), that is grounded on the analysis of the temporal fluctuations of the activity. One evaluates at a given time, $t$, the average membrane potential of the neurons:

$$
A_{N}(t)=\frac{1}{N} \sum_{i=1}^{N} V_{i}(t)
$$

Its time fluctuations can be characterized by the variance

$$
\Delta_{N}=\left\langle A_{N}(t)^{2}\right\rangle_{t}-\left\langle A_{N}(t)\right\rangle_{t}^{2}
$$

This variance is normalized to the population averaged variance of single cell activity

$$
\Delta=\frac{1}{N} \sum_{i=1}^{N}\left(\left\langle V_{i}(t)^{2}\right\rangle_{t}-\left\langle V_{i}(t)\right\rangle_{t}^{2}\right)
$$

The resulting coherence parameter

$$
\Sigma_{N}=\frac{\Delta_{N}}{\Delta}
$$

behaves generally for large $N$ as

$$
\Sigma_{N}=\chi+\frac{a}{N}+O\left(\frac{1}{N^{2}}\right)
$$

where $a$ is some constant number and $\chi$, comprised between 0 and 1 , measures the degree of coherence in the system. In particular, $\chi=1$ if the system is totally synchronized (i.e., $V_{i}(t)=V(t)$ for all $i$ ) and $\chi=0$ if the state of the system is asynchronous.

\section{References}

Abbott L.F. and van Vreeswijk C. (1993). Asynchronous states in networks of pulse-coupled oscillators. Phys. Rev. E48, 1483-1490. 
Ahmed B., Anderson J. C., Douglas R. J., Martin K. A., and Whitteridge D. (1998). Cereb. Cortex 8:462-76.

Butera, R.J. Jr, Rinzel, J., and Smith, J.C. (1999). Models of respiratory rhythm generation in the Pre-Botzinger Complex. II. Populations of coupled pacemaker neurons. J. Neurophysiol., 82:398-415.

Cattaneo, B., Maffei, L., and Morrone, C. (1981). Patterns in the discharge of simple and complex visual cortical cells. Proc. R. Soc. Lond. B Biol. Sci., 212, 279-297.

Chow, C. (1998). Phaselocking in weakly heterogeneous neural networks. Physica $D$, 118:343-370.

Connors, B.W. and Gutnick, M.J. (1990). Intrinsic firing patterns of diverse neocortical neurons. Trends. Neurosci. 13:99-104.

Connors, B.W., Gutnick, M.J., and Prince, D.A. (1982) Electrophysiological properties of neocortical neurons in vitro. J. Neurophysiol., 48:1302-1320.

Crook, S.M., Ermentrout, G.B., and Bower, J.M. (1998). Spike frequency adaptation affects the synchronization properties of networks of cortical oscillations. Neural Comput 10, 837-54.

DeBusk, B., DeBruyn, E., Snider, R., Kabara, J., and Bonds, A. (1997). Stimulusdependent modulation of spike burst length in cat striate cortical cells, J. Neurophysiol. 78, 199-213.

Ermentrout, E. (1994). Reduction of conductance-based models with slow synapses to neural nets. Neural Comput. 6, 679-695.

Flint, A.C., and Connors B.W. (1996). Two types of network oscillations in neocortex mediated by distinct glutamate receptor subtypes and neuronal populations. J. Neurophysiol. 75 951-957.

Gerstner, W., van Hemmen, J.L., and Cowan, J. (1996). "What matters in neuronal locking", Neural Comput. 8, 1653-1676.

Golomb, D. and Amitai, Y. (1997) Propagating neuronal discharges in neocortical slices: Computational and experimental study J. Neurophysiol. 78 1199-1211.

Golomb, D. and Hansel D. (1999) The number of synaptic inputs and the synchrony of large sparse neuronal networks, Neural Computation, in press.

Gray C.M. (1994). Synchronous oscillations in neuronal systems: mechanisms and functions. J. Comp. Neurosci. 1, 11-38. 
Gutnick, M.J., Connors, B.W., and Prince, D.A. (1982) Mechanisms of neocortical epileptogenesis in vitro. J. Neurophysiol. 48 1321-1335.

Hansel D., Mato G. and Meunier C. (1993). Phase reduction and neural modeling. in Functional Analysis of the brain based on multiple-site recordings, Concepts in Neuroscience 4, 192-210.

Hansel D., Mato G. and Meunier C. (1995). Synchrony in excitatory neural networks. Neural Comput. 7, 307-337.

Hansel D., Mato G., Meunier C., and Neltner, L. (1998). On numerical simulations of integrate-and-fire neural networks. Neural Comput. 10, 467-485.

Hansel, D. and Sompolinsky, H. (1998). Modeling feature selectivity in local cortical circuits. In Methods in Neuronal Modeling. ¿From Synapses to Networks, C. Koch, and I. Segev, second edition. MIT Press, Cambridge, MA.

Hubel, D., (1959) Single unit activity in striate cortex of unrestrained cats, $J$. Physiol. (London), 147, 226-238.

Latham, P.E., Richmond, B.J., Nelson, P.G., and Nirenberg, S. (1999) Intrinsic dynamics in neural networks. I. Theory. J. Neurophysiol. in press.

Lapicque, L. (1907). Recherches quantitatives sur l'excitation électrique des nerfs traitée comme une polarisation. J. Physiol. Pathol. Gen, 9, 620-635.

Li, Z. and Dayan, P. (1999). Computational differences between asymmetrical and symmetrical networks. Network 10, 59-78.

Neltner, L., Hansel, D., Mato, G., and Meunier, C. (1999). Synchrony in heterogeneous networks of spiking neurons, Neural Computation, in press.

O’Donovan, M.J., Wenner, P., Chub, N., Tabak, J., and Rinzel, J. (1998). Mechanisms of spontaneous activity in the developing spinal cord and their relevance to locomotion. Ann. N.Y. Acad. Sci. 860, 130-141.

Pinsky P.F. and Rinzel J. (1994). Intrinsic and network rhythmogenesis in a reduced Traub model for CA3 neurons. J. Comput. Neurosci., 1, 39-60.

Rinzel, J. and Ermentrout, G. B. (1998). Analysis of Neural Excitability and Oscillations. In Methods in Neuronal Modeling. ¿From Synapses to Networks, C. Koch, and I. Segev, second edition. MIT Press, Cambridge, MA.

Shelley, M. (1999). Efficient and accurate integration schemes for systems of integrate-and-fire neurons. Preprint. 
Shriki, O., Hansel, D. and Sompolinsky, H. (1999). Rate Models for Conductance Based Cortical Neuronal Networks, submitted.

Silva, L.R., Amitai, Y., and Connors, B.W. (1991). Intrinsic oscillations of neocortex generated by layer pyramidal neurons. Science 251 432-435.

Snider, R., Kabara, J., Roig, B. and Bonds, A., (1998). Burst firing and modulation of functional connectivity in cat striate cortex, J. Neurophysiol 80, 730-744.

Steriade, M., McCormick, D.A., and Sejnowski, T.J. (1983). Thalamocortical oscillations in the sleeping and aroused brain. Science 262, 679-685.

Traub R.D., Miles R. and Buzsaki G. (1992). Computer simulation of carbacholdriven rhythmic population oscillations in the CA3 region of the in vitro rat hippocampus. J. Physiol. (Lond), 51, 653-72.

Tuckwell H.C. (1988). Introduction to theoretical neurobiology, Cambridge University Press, New York.

van Vreeswijk, C. (1996). Partial synchronization in populations of pulse-coupled oscillators, Phys. Rev. E 54, 5522-5537

van Vreeswijk, C. (1999), Analysis of the asynchronous state in networks of strongly coupled oscillators, in preparation.

van Vreeswijk, C., Abbott, L.F., and Ermentrout, G.B. (1994). When inhibition not excitation synchronizes neural firing. J. Comput. Neurosci. 1, 313-321.

White, J.A., Chow, C.C., Ritt, J., Soto-Treviño, C., and Kopell, N. (1998). Synchronous oscillations in heterogeneous, mutually inhibited neurons. J. Comput. Neurosci. 5, 5-16.

Whittington, M.A., Traub, R.D, and Jeffreys, J.G.R. (1995). Synchronized oscillations in interneuron networks driven by metabotropic glutamate receptor activation. Nature 373, 612-615.

Wilson, H.R and Cowan, J.D. (1972). Excitatory and inhibitory interactions in localized populations of model neurons. Biophys. J. 12, 1-24. 